\title{
Chiral symmetry breaking with no bilinear condensate revisited
}

\author{
Takuya Kanazawa \\ iTHES Research Group and Quantum Hadron Physics Laboratory, RIKEN, \\ Wako, Saitama 351-0198, Japan \\ E-mail: takuya.kanazawa@riken.jp
}

ABSTRACT: While chiral symmetry breaking in the QCD vacuum is attributed to nonzero chiral condensate, an alternative symmetry breaking pattern with no chiral condensate is also possible, as pointed out by Stern. This hypothetical phase was excluded in QCD at zero density long time ago, but nothing forbids it at finite baryon density. In this work, we study the $\theta$ dependence of this unorthodox phase on the basis of chiral perturbation theory. Physical observables such as energy density, topological susceptibility, non-local chiral order parameter and meson masses are computed analytically in the $\varepsilon$-regime. At nonzero $\theta$ we find an exotic phase that breaks vectorial flavor symmetries in a way analogous to the Aoki phase in lattice QCD.

KEYwords: Spontaneous Symmetry Breaking, Chiral Lagrangians, Effective field theories, Phase Diagram of QCD

ARXIV EPRINT: 1507.06376 


\section{Contents}

1 Introduction 1

2 Chiral effective theory 3

2.1 Symmetries and effective Lagrangian 3

2.1.1 Massless quarks 3

2.1.2 Massive quarks 5

$\begin{array}{lll}2.2 & \text { Finite-volume partition function } & 7\end{array}$

3 The $\boldsymbol{\theta}$ vacua $r$

3.1 Quarks in the fundamental representation $\quad 11$

$\begin{array}{ll}3.2 & \text { Quarks in higher representations } \\ \end{array}$

$\begin{array}{lll}4 & \text { Conclusion } & 19\end{array}$

$\begin{array}{ll}\text { A Derivation of } \chi_{u d} \text { in }(2.24) & 20\end{array}$

\section{Introduction}

Spontaneous symmetry breaking can be characterized by order parameters that transform nontrivially under the symmetry of interest. A commonly used order parameter for chiral symmetry breaking in Quantum Chromodynamics (QCD) is the chiral condensate, $\langle\bar{\psi} \psi\rangle$. It is linked to the accumulation of near-zero Dirac eigenvalues through the Banks-Casher relation [1]. Another order parameter is the pion decay constant, $F_{\pi}$. Some time ago Stern $[2,3]$ pointed out that the condition for $F_{\pi} \neq 0$ is weaker than that for $\langle\bar{\psi} \psi\rangle \neq 0$, suggesting the possibility of an exotic phase in QCD in which $\langle\bar{\psi} \psi\rangle=0$ but $F_{\pi} \neq 0 .{ }^{1}$ We will refer to this phase as the Stern phase. Chiral symmetry breaking in this phase could be triggered by four-quark condensates such as $\left\langle\bar{\psi} \lambda^{a} \gamma_{\mu}\left(1-\gamma_{5}\right) \psi \cdot \bar{\psi} \lambda^{a} \gamma_{\mu}\left(1+\gamma_{5}\right) \psi\right\rangle$ and $\left\langle\bar{\psi} \lambda^{a}\left(1-\gamma_{5}\right) \psi \cdot \bar{\psi} \lambda^{a}\left(1+\gamma_{5}\right) \psi\right\rangle$, with $\left\{\lambda^{a}\right\}$ the flavor generators [4]. ${ }^{2}$ These condensates leave the discrete anomaly-free subgroup of $\mathrm{U}(1)_{A}$ unbroken, which ensures a vanishing chiral condensate. (Actually the possibility of an unbroken discrete axial symmetry was pointed out by Dashen long time ago [6].) The Stern phase is analogous to antiferromagnets which has no global magnetization; also similar is the so-called molecular Bose-Einstein condensation (BEC) $[7,8]$ which is distinguished from the atomic BEC by an unbroken $\mathbb{Z}_{2}$ symmetry.

\footnotetext{
${ }^{1}$ Other bilinear condensates such as $\left\langle\bar{\psi} T^{a} G_{\mu \nu}^{a} \sigma_{\mu \nu} \psi\right\rangle$ are assumed to vanish as well.

${ }^{2}$ While quartic condensates also form in color-superconducting phases of QCD at high density [5], the baryon number symmetry $\mathrm{U}(1)_{B}$ is not broken in the Stern phase. Moreover the patterns of $\mathrm{U}(1)_{A}$ symmetry breaking in the Stern phase and in the color-superconducting phases are different, as will be explained later.
} 
Soon after the proposal, the Stern phase in QCD was critically examined in [4] where it was proved with rigorous QCD inequalities that this phase is ruled out in QCD at any temperature and zero density. This proof, however, leaves open the possibility that the Stern phase may emerge in QCD at nonzero chemical potential since the complex pathintegral measure invalidates the use of QCD inequalities. Indeed, a Ginzburg-Landau-type analysis in a chiral effective model suggests that this is likely to be the case [9]. In addition, studies of inhomogeneous chirally broken phases in dense QCD suggest that chiral symmetry breaking in such phases could be driven not by the chiral condensate but rather by a higher-order condensate $[10,11]$ : the basic idea of [11] is that a one-dimensionally modulated chiral condensate is wiped out by thermal fluctuations of phonons, whereas [10] shows in QCD at large $N$ that a higher-order chiral order parameter whose spatial average is nonzero must exist when the chiral condensate is locally nonzero but its spatial average vanishes. Recently, phases with massive fermions with no bilinear condensate have been found in numerical simulations [12, 13], which bears resemblance to the Stern phase. So, even though the presence of the Stern phase in QCD remains an open problem for now, we have pieces of circumstantial evidence suggesting that the Stern phase is a realistic possibility worthy of serious consideration. This will be of importance for our better understanding of the QCD phase diagram, which is still only poorly understood [5, 14].

If the Stern phase indeed exists in the finite-density QCD, there must be a transition from a hadronic phase to the Stern phase as $\mu$ is varied. A possible phase structure at $\mu \neq 0$ was proposed in [9] where two transitions were reported along the $\mu$ axis: from the hadronic phase to the Stern phase and then to the chirally symmetric phase. These can become smooth crossovers for nonzero quark masses. At the first transition the chiral condensate drops dramatically while the four-quark condensate is unaffected. A rapid rise of the baryon number susceptibility is a signal of this transition. We would also like to mention another scenario based on the idea of inhomogeneous condensation [11] in which the above transitions are both second order in the chiral limit; at the low- $\mu$ transition, it is a proliferation of domain walls and associated Nambu-Goldstone modes that drive the chiral condensate to zero. These pictures are based on effective models and a quantitative precision is not expected, but nonetheless their symmetry-based arguments are robust predictions that can be tested in QCD-based calculations in future.

In this paper, we investigate various aspects of low-energy physics in the Stern phase by means of chiral perturbation theory. In particular the structure of the $\theta$ vacua in the Stern phase is analyzed in great details for the first time. We find behaviors that differ drastically from those in the orthodox $\theta$ vacuum. The competition between multiple leading terms in the chiral Lagrangian is shown to lead to a nontrivial phase diagram at nonzero $\theta$. Not only quarks in the fundamental representation of the gauge group, but also those in higher representations are considered and new results are obtained.

This paper is organized as follows. In section 2 we sort out the breaking pattern of continuous and discrete symmetries in the Stern phase and present a systematic derivation of the chiral effective theory. While this part overlaps with preceding works $[4,15,16]$, we extend them by considering the most general breaking pattern of the discrete axial symmetry. One of the new results here is the existence of topologically stable domain walls 
in the Stern phase, and another is an analytical calculation of the volume dependence of a non-local chiral order parameter in the $\varepsilon$-regime. In section 3 we introduce the $\theta$ angle into the low-energy effective theory and compute various observables such as energy density, topological susceptibility, topological density and pion masses. It is revealed that lowenergy physics at $\theta \neq 0$ is sensitive to a subtle balance between leading terms in the chiral Lagrangian. An exotic phase similar to the Aoki phase of Wilson fermions [17] is uncovered, and its domain of existence is determined in the phase diagram. Finally we consider QCD with quarks in higher representations and elucidate a multi-branched $\theta$ dependence of the energy density that surprisingly differs from the case of fundamental quarks. We conclude in section 4.

\section{Chiral effective theory}

\subsection{Symmetries and effective Lagrangian}

In this section we classify low-energy chiral effective theories for the Stern phase, generalizing preceding works in $[4,9,15,16]$. We will also give a brief account of topologically stable domain walls in the Stern phase, which has not been discussed to date.

\subsubsection{Massless quarks}

Let us consider $\mathrm{SU}(N)$ gauge theory with $N_{f} \geq 2$ massless Dirac fermions in a complex representation $R$ of $\mathrm{SU}(N)$ in Euclidean spacetime. As is well known, the classical $\mathrm{U}(1)_{A}$ symmetry in the chiral limit is violated by quantum effects due to instantons $[18,19]$, but generally there exists a discrete remnant of the $\mathrm{U}(1)_{A}$ symmetry. According to the index theorem, the index $I_{R}$ of the Dirac operator in the representation $R$ for a single instanton background is given by $[20,21]$

$$
I_{R}=2 T_{R},
$$

with $T_{R}$ defined by $\operatorname{tr}\left(T^{a} T^{b}\right)=T_{R} \delta^{a b}$ for $\mathrm{SU}(N)$ generators in the representation $R$; e.g.,

$$
T_{\text {Fund }}=\frac{1}{2}, \quad T_{\text {Adj }}=N, \quad T_{\mathrm{S}}=\frac{N+2}{2} \quad \text { and } \quad T_{\mathrm{AS}}=\frac{N-2}{2},
$$

where $\mathrm{S}(\mathrm{AS})$ stands for the two-index symmetric (anti-symmetric) representation of $\mathrm{SU}(N)$, respectively. This implies that the $\mathrm{U}(1)_{A}$ symmetry shrinks to $\mathbb{Z}_{4 N_{f} T_{R}}$ due to quantum effects. Then the orthodox pattern of chiral symmetry breaking with $\langle\bar{\psi} \psi\rangle \neq 0 \operatorname{reads}^{3}$

$$
\mathrm{SU}\left(N_{f}\right)_{R} \times \mathrm{SU}\left(N_{f}\right)_{L} \times \mathrm{U}(1)_{B} \times\left(\mathbb{Z}_{4 N_{f} T_{R}}\right)_{A} \longrightarrow \mathrm{SU}\left(N_{f}\right)_{V} \times \mathrm{U}(1)_{B} .
$$

By contrast, the putative Stern phase entails a different pattern of chiral symmetry breaking:

$$
\mathrm{SU}\left(N_{f}\right)_{R} \times \mathrm{SU}\left(N_{f}\right)_{L} \times \mathrm{U}(1)_{B} \times\left(\mathbb{Z}_{4 N_{f} T_{R}}\right)_{A} \longrightarrow \mathrm{SU}\left(N_{f}\right)_{V} \times \mathrm{U}(1)_{B} \times\left(\mathbb{Z}_{K}\right)_{A},
$$

\footnotetext{
${ }^{3}$ Precisely speaking, $\mathbb{Z}_{2 N_{f}} \subset \mathbb{Z}_{4 N_{f} T_{R}}$ is part of the $\mathrm{SU}\left(N_{f}\right)_{R} \times \mathrm{SU}\left(N_{f}\right)_{L} \times \mathrm{U}(1)_{B}$ group and has to be factored out in the l.h.s. of (2.3) to avoid double counting. For the same reason, $\mathbb{Z}_{N_{f}}$ which is contained in both $\mathrm{SU}\left(N_{f}\right)_{V}$ and $\mathrm{U}(1)_{B}$ has to be factored out in the r.h.s. of (2.3). However we shall be cavalier on these formalities for simplicity of exposition.
} 
where $2<K \leq 4 N_{f} T_{R}$ is an even divisor of $4 N_{f} T_{R} \cdot{ }^{4}$ (Note that, when $K=2,(2.4)$ is equivalent to (2.3) and there is nothing new.) While only the specific case of $K=$ $4 N_{f} T_{\text {Fund }}=2 N_{f}$ has been discussed in the literature $[4,9,16]$, the other values of $K$ are also theoretically admissible. As the residual $\left(\mathbb{Z}_{K}\right)_{A}$ with $K>2$ enforces $\langle\bar{\psi} \psi\rangle=$ 0 , chiral symmetry breaking in the Stern phase must be driven by higher-dimensional condensates [4]. For example, $\left\langle\left(\bar{\psi}_{R} \psi_{L}\right)^{n}\right\rangle+$ h.c. $\neq 0$ for some $n \geq 2$ corresponds to $K=2 n$, whereas $\left\langle\bar{\psi}_{R} \psi_{L} \cdot \bar{\psi}_{L} \psi_{R}\right\rangle \neq 0$ or $\left\langle\left[\operatorname{det}\left(\bar{\psi}_{R}^{f} \psi_{L}^{g}\right)\right]^{2 T_{R}}\right\rangle \neq 0$ (this is nothing but the 't Hooft vertex) corresponds to the maximal unbroken symmetry, $K=4 N_{f} T_{R}$.

The vacuum structure of the Stern phase must be understood with some care. Since $\left(\mathbb{Z}_{4 N_{f} T_{R}}\right)_{A}$ is spontaneously broken to $\left(\mathbb{Z}_{K}\right)_{A}$, it appears at first sight that there will be $4 N_{f} T_{R} / K$ isolated degenerate vacua. This is not quite correct, however. The point is that two vacua that can be rotated to each other via an action of $\left(\mathbb{Z}_{2 N_{f}}\right)_{A}$ are not isolated, but are continuously connected to each other with no potential barrier via a non-Abelian chiral transformation. This is obvious from the fact that $\left(\mathbb{Z}_{N_{f}}\right)_{R} \times\left(\mathbb{Z}_{N_{f}}\right)_{L} \subset \mathrm{SU}\left(N_{f}\right)_{R} \times \mathrm{SU}\left(N_{f}\right)_{L}$. As a result, the would-be domain walls separating such vacua are unstable, as stressed in [22] for a fractional axial domain wall in the QCD vacuum.

Then, under what conditions does a stable domain wall exist in the Stern phase? Evidently there must be multiple vacua that cannot be rotated to each other via a combined action of $\left(\mathbb{Z}_{K}\right)_{A}$ and $\left(\mathbb{Z}_{2 N_{f}}\right)_{A}$. It is not difficult to see that this is true if and only if

$$
\operatorname{LCM}\left(K, 2 N_{f}\right)<4 N_{f} T_{R},
$$

where $\operatorname{LCM}(a, b)$ for $a, b \in \mathbb{N}$ is the least common multiple of $a$ and $b$. We also see that

$$
\left(\begin{array}{c}
\text { the number of disconnected components } \\
\text { of the vacuum manifold }
\end{array}\right)=\frac{4 N_{f} T_{R}}{\operatorname{LCM}\left(K, 2 N_{f}\right)} .
$$

The bottom line is that a stable domain wall can exist under the condition (2.5) and that the variety of domain walls is determined by (2.6). Let us make a few quick comments. First, the r.h.s. of (2.6) is always a positive integer because both $K$ and $2 N_{f}$ are divisors of $4 N_{f} T_{R}$. Secondly, (2.5) cannot be satisfied if $T_{R}=1 / 2$, implying that the vacuum manifold is connected for fundamental quarks for any $K$. Thirdly, when $K=2$ (i.e., the QCD vacuum with chiral condensate), the number of isolated vacua is given by $2 T_{R}$, as follows from (2.6).

Let us comment on the literature. It is well known that in $\mathcal{N}=1 \mathrm{SU}(N)$ Super-YangMills (SYM) theory, $\mathbb{Z}_{2 N} \subset \mathrm{U}(1)_{R}$ breaks down spontaneously to $\mathbb{Z}_{2}$ through gaugino condensation $[23,24]$. There are $N$ isolated ground states that are discriminated by phases of the condensate as $\langle\lambda \lambda\rangle \sim \Lambda^{3} \exp (2 \pi i k / N)$ with $k=0,1, \ldots, N-1$, and stable domain walls exist [25-27]. We also wish to mention the so-called axion domain walls [28], which have been discussed widely in axion cosmology. There is an apparent similarity between domain walls in these theories and those in the Stern phase, and we anticipate that many properties would be shared in common. Nevertheless, it deserves attention that the Stern

\footnotetext{
${ }^{4} K$ should be even because $\left(\mathbb{Z}_{2}\right)_{A}$ that flips the sign of $\psi$ is just a $2 \pi$ rotation of space, which cannot be spontaneously broken when the Lorentz symmetry is intact.
} 
phase possesses gapless pion excitations, which are missing in SYM and the axion theory. It would be intriguing to explore physical consequences of this difference in details.

\subsubsection{Massive quarks}

Next we switch on the mass term $\bar{\psi}_{L} M \psi_{R}+\bar{\psi}_{R} M^{\dagger} \psi_{L}$ in the microscopic Lagrangian, with $M$ the $N_{f} \times N_{f}$ quark mass matrix. This term breaks $\left(\mathbb{Z}_{K}\right)_{A}$ down to $\left(\mathbb{Z}_{2}\right)_{A}$ explicitly. However, one can make the Lagrangian invariant under $\left(\mathbb{Z}_{K}\right)_{A}$ if $M$ along with $\psi_{R / L}$ transform as

$$
\psi_{R} \rightarrow \mathrm{e}^{i \phi} \psi_{R}, \quad \psi_{L} \rightarrow \mathrm{e}^{-i \phi} \psi_{L} \quad \text { and } \quad M \rightarrow \mathrm{e}^{-2 i \phi} M \quad \text { for } \quad \mathrm{e}^{i \phi} \in\left(\mathbb{Z}_{K}\right)_{A} .
$$

This symmetry should be preserved in the low-energy effective Lagrangian $\mathcal{L}(U, M)$ of the $N_{f}^{2}-1$ Nambu-Goldstone modes, pions, denoted collectively by $U(x)$. Since the axial current $A_{\mu}^{a}=\bar{\psi} \gamma_{\mu} \gamma_{5} \lambda^{a} \psi$ is neutral under $\left(\mathbb{Z}_{K}\right)_{A}$, so are pions. Thus the condition on the effective theory imposed by $\left(\mathbb{Z}_{K}\right)_{A}$ invariance reads

$$
\mathcal{L}(U, M)=\mathcal{L}(U, z M) \quad \text { for } \quad{ }^{\forall} z \in \mathbb{Z}_{K / 2} .
$$

Using the $N_{f} \times N_{f}$ coset variable $U \in \mathrm{SU}\left(N_{f}\right)_{A}$ as a building block, one can straightforwardly write down the most general chiral Lagrangian consistent with (2.8) [16]. Let us start the classification with $K=4$ and $N_{f}>2$. Then all the odd powers of $M$ are forbidden by (2.8) and we find, up to second order in $\partial$ and $M$,

$$
\begin{aligned}
\mathcal{L}_{N_{f}>2}^{K=4}(U, M)= & \frac{f^{2}}{4} \operatorname{tr}\left(\partial_{4} U^{\dagger} \partial_{4} U+v^{2} \partial_{i} U^{\dagger} \partial_{i} U\right)-h|\operatorname{tr}(M U)|^{2} \\
& -\left\{h_{1} \frac{(\operatorname{tr}(M U))^{2}+\operatorname{tr}(M U)^{2}}{2}+\text { h.c. }\right\}-\left\{h_{2} \frac{(\operatorname{tr}(M U))^{2}-\operatorname{tr}(M U)^{2}}{2}+\text { h.c. }\right\} \\
& -g_{1} \operatorname{tr}\left(M M^{\dagger}\right) .
\end{aligned}
$$

The low-energy constants $\left(h, h_{1}, h_{2}\right)$ and the high-energy constant $g_{1}$ are analogous to $L_{6}$, $L_{7}, L_{8}$ and $H_{2}$ in standard chiral perturbation theory at $\mathcal{O}\left(p^{4}\right)$ [29]. They are related to chiral susceptibilities. $h_{1}$ and $h_{2}$ can be complex in general. The pion velocity $v$ can differ from the speed of light owing to the breaking of Lorentz symmetry in medium. We note that $f, v, h, h_{1}, h_{2}$ and $g_{1}$ all depend on the chemical potential $\mu$ implicitly.

For two flavors, the identity $(\operatorname{tr}(M U))^{2}-\operatorname{tr}(M U)^{2}=2 \operatorname{det} M$ allows us to cast the leading-order Lagrangian in the form

$$
\begin{aligned}
\mathcal{L}_{N_{f}=2}^{K=4}(U, M)= & \frac{f^{2}}{4} \operatorname{tr}\left(\partial_{4} U^{\dagger} \partial_{4} U+v^{2} \partial_{i} U^{\dagger} \partial_{i} U\right)-h|\operatorname{tr}(M U)|^{2}-\left\{\tilde{h} \operatorname{tr}(M U)^{2}+\text { h.c. }\right\} \\
& -g_{1} \operatorname{tr}\left(M M^{\dagger}\right)-\left(g_{2} \operatorname{det} M+\text { h.c. }\right) .
\end{aligned}
$$

While the last term is independent of the pion field, it is $\theta$-dependent and contributes to the topological susceptibility (cf. section 3.1). As a side remark we mention that it plays an important role in QCD at high temperature [30].

The absence of the linear term $\operatorname{tr}(M U)+$ h.c. in (2.9) and (2.10) is consistent with $\langle\bar{\psi} \psi\rangle=0$ in the chiral limit. This implies that the power counting in the $p$-regime of this 
phase must be modified from the usual one, $\partial \sim \mathcal{O}(p)$ and $M \sim \mathcal{O}\left(p^{2}\right)$, to $\partial \sim M \sim \mathcal{O}(p) .^{5}$ As for the pion mass, (2.9) and (2.10) imply $m_{\pi} \propto M$ [4], in contradistinction to the conventional picture where $m_{\pi}^{2} \propto M$. Actually the abnormal scaling $m_{\pi} \propto M$ has been known for the color-flavor-locked (CFL) phase of dense QCD [32-34], superfluid phase of dense two-color QCD [35] and two-flavor QCD at $\theta=\pi$ [36-38]. What is common in all these cases is that the chiral condensate is either zero or negligibly small.

The three $\mathcal{O}\left(M^{2}\right)$ contributions in (2.9) have different origins. If the order parameter of symmetry breaking is in the adjoint representation of $\mathrm{SU}\left(N_{f}\right)_{R / L}$, the leading massdependent contribution should be $\operatorname{tr}_{\text {Adj }}(M U)=|\operatorname{tr}(M U)|^{2}-1$. Thus the term $\propto h$ in (2.9) originates from a condensate that transforms in the adjoint flavor representation. Similarly, the term $\propto h_{1}\left(\propto h_{2}\right)$ comes from a condensate in the two-index symmetric (antisymmetric) flavor representation, respectively. ${ }^{6}$

Let us finally consider $K>4 .^{7}$ This time the effective theory is considerably simplified:

$$
\mathcal{L}^{K>4}(U, M)=\frac{f^{2}}{4}\left(\partial_{4} U^{\dagger} \partial_{4} U+v^{2} \partial_{i} U^{\dagger} \partial_{i} U\right)-h|\operatorname{tr}(M U)|^{2}-g_{1} \operatorname{tr}\left(M M^{\dagger}\right)
$$

at leading order. The other $\mathcal{O}\left(M^{2}\right)$ terms are banned by the discrete symmetry (2.8). Consequently, $\mathcal{L}^{K>4}$ enjoys invariance under an arbitrary phase rotation of $M$, which is equivalent to a vanishing topological susceptibility at this order. In other words, topologically nontrivial sectors are entirely suppressed.

The classification of the effective theory [(2.9), (2.10) and (2.12)] for general $K$ is the main result of this subsection.

A brief comment is in order concerning hadrons other than pions. For the effective theory of pions to be a valid low-energy description, the baryon sector must have a mass gap. ${ }^{8}$ However the status of baryons in the Stern phase is still elusive. In principle, dynamical masses of fermions can be generated without bilinear condensate as evidenced in $[12,13]$. Also in QCD, it has been recognized historically that baryons in the "mirror assignment" can acquire a dynamical mass even when the chiral condensate vanishes [4244]. Previous researches on the Stern phase have found that the baryon spectrum in the Stern phase crucially depends on the chirality assignment of baryons [4, 9]. Instead of trying to resolve this delicate issue, we shall content ourselves in this paper by assuming that the baryon sector is fully gapped. It should also be kept in mind that, at high density

\footnotetext{
${ }^{5}$ This is similar to the generalized chiral perturbation theory [31]. Note however that ref. [31] retains nonzero chiral condensate whereas we put it to zero exactly as a consequence of the $\left(\mathbb{Z}_{K}\right)_{A}$ symmetry.

${ }^{6}$ In the CFL phase, the leading gauge-invariant order parameter of chiral symmetry breaking is the four-quark condensate [39, 40]

$$
\left\langle\varepsilon_{f g h} \varepsilon_{i j k} \bar{\psi}_{R}^{f} \bar{\psi}_{R}^{g} \psi_{L}^{i} \psi_{L}^{j}\right\rangle \propto \delta_{h k},
$$

which transforms in the anti-symmetric representation of $\mathrm{SU}(3)_{R / L}$. Reflecting this pattern, the chiral Lagrangian in the CFL phase only contains the term $\propto h_{2}$ in (2.9); $h=h_{1}=0$ is indeed confirmed in explicit microscopic calculations [33].

${ }^{7}$ We note that the condition $K>4$ is consistent with $K \leq 4 N_{f} T_{R}$ only if $N_{f} T_{R}>1$. This means that $R$ must be higher than fundamental for $N_{f}=2$, whereas no such constraint arises for $N_{f}>2$.

${ }^{8}$ Since chiral symmetry is spontaneously broken in the Stern phase, the 't Hooft anomaly matching condition [41] is satisfied by pions. If some baryons happen to be gapless, then it would be highly nontrivial to keep the anomaly matching satisfied. This seems to be a rather unlikely possibility.
} 
or in the large- $N$ limit, instanton effects are suppressed and the pseudo-Nambu-Goldstone mode $\left(\eta^{\prime}\right)$ associated with $\mathrm{U}(1)_{A}$ breaking gets light. Then one has to incorporate $\eta^{\prime}$ into the effective Lagrangian as well. This is an intriguing situation but will not be covered in this paper.

\subsection{Finite-volume partition function}

When pions are sufficiently light in a finite volume, their zero-mode fluctuations become non-perturbative and have to be integrated out exactly. This occurs in the so-called $\varepsilon$ regime $[45,46]$ where the linear extent $L$ of the Euclidean box is such that

$$
\frac{1}{\Lambda_{\mathrm{QCD}}} \ll L \ll \frac{1}{m_{\pi}}
$$

This means that the contribution of hadrons other than pions to the partition function is suppressed (first inequality) whereas pions' Compton length is sufficiently larger than the box size (second inequality) so that the non-zero modes of pions become irrelevant. This regime can be realized by taking the double limits $V_{4}=L^{4} \rightarrow \infty$ and $M \rightarrow 0$ with $V_{4} M^{2} \Lambda_{\mathrm{QCD}}^{2}$ fixed. More formally stated, we shall adopt an exotic $\varepsilon$-expansion scheme with $\partial \sim 1 / L \sim \mathcal{O}(\varepsilon)$ and $M \sim \mathcal{O}\left(\varepsilon^{2}\right)$ [15]. A similar scheme was used in dense QCD [35, 47] but it differs from the conventional $\varepsilon$-expansion with $M \sim \mathcal{O}\left(\varepsilon^{4}\right)$. This disparity of course stems from the absence of the linear mass term in the Stern phase.

In this limiting regime, the QCD path integral reduces to a finite-dimensional integral over pion zero modes. For the three cases in section 2.1 we obtain, respectively, ${ }^{9}$

$$
\begin{aligned}
Z_{N_{f}>2}^{K=4}(M)= & \int_{\mathrm{SU}\left(N_{f}\right)} \mathrm{d} U \exp \left[V _ { 4 } \left\{h|\operatorname{tr}(M U)|^{2}+\left(h_{1} \frac{(\operatorname{tr}(M U))^{2}+\operatorname{tr}(M U)^{2}}{2}+\text { h.c. }\right)\right.\right. \\
& \left.\left.+\left(h_{2} \frac{(\operatorname{tr}(M U))^{2}-\operatorname{tr}(M U)^{2}}{2}+\text { h.c. }\right)+g_{1} \operatorname{tr}\left(M M^{\dagger}\right)\right\}\right], \\
Z_{N_{f}=2}^{K=4}(M)= & \int_{\mathrm{SU}(2)} \mathrm{d} U \exp \left[V _ { 4 } \left\{h|\operatorname{tr}(M U)|^{2}+\left(\tilde{h} \operatorname{tr}(M U)^{2}+\text { h.c. }\right)+g_{1} \operatorname{tr}\left(M M^{\dagger}\right)\right.\right. \\
& \left.\left.\quad+\left(g_{2} \operatorname{det} M+\text { h.c. }\right)\right\}\right], \\
Z^{K>4}(M)= & \int_{\mathrm{SU}\left(N_{f}\right)} \mathrm{d} U \exp \left[V_{4}\left\{h|\operatorname{tr}(M U)|^{2}+g_{1} \operatorname{tr}\left(M M^{\dagger}\right)\right\}\right],
\end{aligned}
$$

where $\mathrm{d} U$ denotes the Haar measure. These expressions give exact mass and volumedependence of the partition function in the $\varepsilon$-regime, which is not only theoretically interesting but also useful in that we can extract physical quantities in the infinite-volume limit (e.g., low energy constants $h, h_{1}$ and $h_{2}$ ) from numerical data obtained in a finite volume

\footnotetext{
${ }^{9} \mathrm{~A}$ similar finite-volume analysis was performed in [15], but the authors did not specify the discrete symmetry responsible for the vanishing chiral condensate, nor did they underline the distinction between $K=4$ and $K>4$ whose importance is clear from (2.14). Quarks in higher representation of the gauge group were not considered, either.
} 
through fitting to finite-volume formulas extracted from (2.14). ${ }^{10}$ We hope that analytical results in this section serve as a guide in future lattice simulations of the Stern phase.

Partition function for $\boldsymbol{K}>\boldsymbol{4}$. Since the structure of the partition functions (2.14) is mathematically more involved than in the conventional $\varepsilon$-regime, we shall focus our attention on the $K>4$ case, $(2.14 \mathrm{c})$, for simplicity. As $\mathrm{SU}\left(N_{f}\right)_{V}$ symmetry is assumed to be unbroken in the Stern phase, we assume that $U=\mathbb{1}$ is the ground state. This fixes the sign of $h$ to positive. Extending the manifold of integration to $\mathrm{U}\left(N_{f}\right)$, we obtain

$$
\begin{aligned}
& \exp \left[-V_{4} g_{1} \operatorname{tr}\left(M M^{\dagger}\right)\right] Z^{K>4}\left(\left\{m_{f}\right\}\right) \\
& =\int_{\mathrm{U}\left(N_{f}\right)} \mathrm{d} U \exp \left[V_{4} h \operatorname{tr}(M U) \operatorname{tr}\left(U^{\dagger} M^{\dagger}\right)\right] \\
& =\frac{1}{\pi} \int_{\mathbb{C}} \mathrm{d}^{2} z \mathrm{e}^{-|z|^{2}} \int_{\mathrm{U}\left(N_{f}\right)} \mathrm{d} U \exp \left[z \sqrt{V_{4} h} \operatorname{tr}(M U)+z^{*} \sqrt{V_{4} h} \operatorname{tr}\left(U^{\dagger} M^{\dagger}\right)\right] \\
& =2 \int_{0}^{\infty} \mathrm{d} x x \mathrm{e}^{-x^{2}} \int_{\mathrm{U}\left(N_{f}\right)} \mathrm{d} U \exp \left[2 x \sqrt{V_{4} h} \operatorname{Retr}(M U)\right] .
\end{aligned}
$$

In the last step the phase of $z$ was absorbed in $U$. We now set $M=\operatorname{diag}\left(m_{f}\right)$ and define $\mu_{f} \equiv 2 \sqrt{V_{4} h} m_{f}$. Assuming ${ }^{\forall} \mu_{f} \in \mathbb{R}$ we substitute the well-known analytic formula for the above unitary integral [49-51] to obtain

$$
Z^{K>4}\left(\left\{\mu_{f}\right\}\right)=C_{N_{f}} \exp \left(\frac{g_{1}}{4 h} \sum_{f} \mu_{f}^{2}\right) \int_{0}^{\infty} \mathrm{d} x x \mathrm{e}^{-x^{2}} \frac{\operatorname{det}_{1 \leq i, j \leq N_{f}}\left[\left(x \mu_{i}\right)^{j-1} I_{j-1}\left(x \mu_{i}\right)\right]}{\Delta\left(\left(x \mu_{1}\right)^{2}, \ldots,\left(x \mu_{N_{f}}\right)^{2}\right)}
$$

where $\Delta\left(a_{1}^{2}, \ldots, a_{N}^{2}\right) \equiv \prod_{i>j}\left(a_{i}^{2}-a_{j}^{2}\right)$ is the Vandermonde determinant, and the normalization constant $C_{N_{f}} \equiv 2^{N_{f}\left(N_{f}-1\right) / 2+1}\left[\prod_{k=1}^{N_{f}-1} k !\right]$ ensures $Z^{K>4} \rightarrow 1$ in the chiral limit. In particular, for $N_{f}=2$ and $\mu_{1}=\mu_{2} \equiv \mu$ we have

$$
Z^{K>4}(\{\mu, \mu\})=\exp \left(\frac{g_{1}+h}{2 h} \mu^{2}\right)\left\{I_{0}\left(\frac{\mu^{2}}{2}\right)-I_{1}\left(\frac{\mu^{2}}{2}\right)\right\} .
$$

Equations (2.18) and (2.19) are new results. Let us contrast (2.19) with the $N_{f}=2$ partition function in the topologically trivial sector of the conventional $\varepsilon$-regime [46]:

$$
Z_{\nu=0}(\{m, m\})=I_{0}^{2}\left(V_{4} \Sigma m\right)-I_{1}^{2}\left(V_{4} \Sigma m\right) .
$$

Chiral susceptibility. Although chiral condensate in the Stern phase vanishes in the chiral limit, there is a non-local order parameter for chiral symmetry breaking. Considering

\footnotetext{
${ }^{10}$ We refer the interested reader to [48] for a review of other miscellaneous intriguing aspects of QCD in a finite volume.
} 


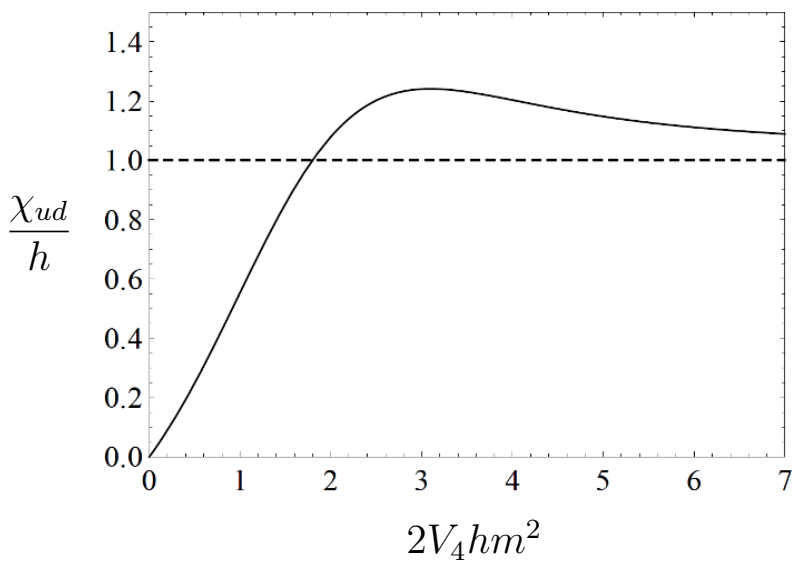

Figure 1. The non-local order parameter (2.21) in the microscopic limit for $K>4$ and $N_{f}=2$ with equal masses.

$N_{f}=2$ for simplicity, we define the disconnected chiral susceptibility

$$
\begin{aligned}
\chi_{u d}(m) & \equiv \int \mathrm{d}^{4} x\left[\left\langle\bar{u}_{L} u_{R}(x) \bar{d}_{R} d_{L}(0)\right\rangle-\left\langle\bar{u}_{L} u_{R}\right\rangle\left\langle\bar{d}_{R} d_{L}\right\rangle\right]+\text { h.c. } \\
& =\lim _{m_{u, d} \rightarrow m} \frac{1}{V_{4}} \frac{\partial^{2}}{\partial m_{u} \partial m_{d}^{*}} \log Z+\text { h.c. }
\end{aligned}
$$

which is singlet under a vectorial isospin rotation but is charged under the axial isospin rotation generated by $\gamma_{5} \tau_{3}$. Thus $\chi_{u d} \neq 0$ in the chiral limit is a signal of spontaneous breaking of $\mathrm{SU}(2)_{A}$. Noting that $g_{1} \operatorname{tr}\left(M M^{\dagger}\right)=g_{1}\left(m_{u} m_{u}^{*}+m_{d} m_{d}^{*}\right)$ gives no contribution to $\chi_{u d}$, we have, for $K>4$ in the $\varepsilon$-regime

$$
\chi_{u d}(m)=\lim _{m_{u, d} \rightarrow m} \frac{1}{V_{4}} \frac{\partial^{2}}{\partial m_{u} \partial m_{d}^{*}} \log \left(\int_{\mathrm{SU}(2)} \mathrm{d} U \exp \left[V_{4} h \operatorname{tr}(M U) \operatorname{tr}\left(U^{\dagger} M^{\dagger}\right)\right]\right)+\text { h.c. }
$$

This expression can be evaluated analytically. After a tedious calculation one finds

$$
\chi_{u d}=h\left[\frac{2}{3} \frac{I_{1}-I_{2}}{I_{0}-I_{1}}+\frac{\mu^{2}}{6} \frac{I_{0}+I_{1}-I_{2}-I_{3}}{I_{0}-I_{1}}-\frac{\mu^{2}}{8}\left(\frac{I_{0}-I_{2}}{I_{0}-I_{1}}\right)^{2}\right],
$$

where $I_{n}$ 's are the modified Bessel functions of the first kind, $I_{n}(x)$, evaluated at $x=\mu^{2} / 2$. The derivation of (2.24) is lengthy and is relegated to appendix A. Figure 1 shows $\chi_{u d}$ as a function of $\mu^{2} / 2=2 V_{4} h m^{2}$. Asymptotically $\chi_{u d}$ behaves as

$$
\frac{\chi_{u d}}{h} \sim \frac{5}{24} \mu^{2} \quad \text { for } \mu \ll 1 \quad \text { and } \quad \frac{\chi_{u d}}{h} \sim 1+\frac{1}{\mu^{2}} \quad \text { for } \mu \gg 1 .
$$

The vanishing of $\chi_{u d}$ for $\mu \rightarrow 0$ is indicative of symmetry restoration at finite volume. In the opposite limit $\mu \gg 1, \chi_{u d}$ approaches $h$, so $h \neq 0$ is in fact an order parameter of chiral symmetry breaking, much like $\Sigma$ in the conventional $\varepsilon$-regime. It is quite intriguing that the behavior of $\chi_{u d}$ in figure 1 is non-monotonic: it approaches $h$ from above! This feature is not seen in the volume dependence of chiral condensate in the QCD vacuum [45]. 
Spectral sum rules. The mass dependence of the partition function also provides detailed information on the statistical distribution of Dirac eigenvalues. Let us first observe that the QCD partition function in the topologically trivial sector may be cast in the form

$$
Z_{\mathrm{QCD}}=\left\langle\prod_{n}^{\prime} \operatorname{det}\left(\mathbb{1}+\frac{M^{\dagger} M}{\lambda_{n}^{2}}\right)\right\rangle_{N_{f}},
$$

where $\left\{i \lambda_{n}\right\}_{n}$ denotes eigenvalues of the Euclidean Dirac operator and the primed product runs over eigenvalues with $\operatorname{Re} \lambda_{n}>0$. The bracket $\langle O\rangle_{N_{f}}$ represents the expectation value of $O$ with the weight of $\mathrm{QCD}$ with $N_{f}$ massless flavors. By expanding $Z_{\mathrm{QCD}}$ in $M M^{\dagger}$ and equating the coefficients with those from the effective theories (2.14a), (2.14b) and $(2.14 \mathrm{c})$, one obtains an infinitely many spectral sum rules obeyed by Dirac eigenvalues. ${ }^{11}$ When $K>4$, the spectral sums generally depend on both $g_{1}$ and $h$. However, the term $g_{1} \operatorname{tr}\left(M M^{\dagger}\right)$ appears even in a free theory and has no bearing on symmetry breaking at low energy. Rather, it serves to absorb UV divergences arising from large perturbative Dirac eigenvalues $[15,52]$. Therefore we should make suitable combinations of spectral sum rules in such a way that $g_{1}$ does not appear explicitly. In terms of rescaled dimensionless Dirac eigenvalues $\zeta_{n} \equiv 2 \sqrt{V_{4} h} \lambda_{n}$, the first few sum rules obtained this way for $K>4$ read

$$
\begin{array}{r}
\left\langle\sum_{n}^{\prime} \frac{1}{\zeta_{n}^{4}}\right\rangle_{N_{f}}=\frac{1}{8 N_{f}\left(N_{f}^{2}-1\right)}, \\
\left\langle\left(\sum_{n}^{\prime} \frac{1}{\zeta_{n}^{2}}-\left\langle\sum_{n}^{\prime} \frac{1}{\zeta_{n}^{2}}\right\rangle_{N_{f}}\right)^{2}\right\rangle_{N_{f}}=\frac{N_{f}^{2}+1}{16 N_{f}^{2}\left(N_{f}^{2}-1\right)} .
\end{array}
$$

The existence of such nontrivial correlations on the scale $\zeta_{n} \sim \mathcal{O}(1)$ suggests that the typical scale of Dirac eigenvalues pertinent to symmetry breaking in the Stern phase is $\sim 1 / \sqrt{V_{4} h}$. This volume dependence is exactly in accord with the prediction by Stern $[2,3]$. While this is in contrast to the conventional microscopic domain of the QCD vacuum where $\lambda_{n} \sim 1 / V_{4} \Sigma$, there is a similarity to the microscopic domain in high-density QCD where $\lambda_{n} \sim 1 / \sqrt{V_{4} \Delta^{2}}$ with $\Delta$ the BCS gap of quarks near the Fermi surface [53], indicating a natural correspondence $\Delta^{2} \leftrightarrow h$. Finally we point out that the reality of the spectral sums (2.27) is rather nontrivial, because the Dirac operator is not assumed to be antiHermitian and eigenvalues $\zeta_{n}$ are complex-valued in general. This may be pointing to a hidden symmetry in the Dirac spectra of the Stern phase.

\section{The $\theta$ vacua}

Physics of the $\theta$ vacuum in QCD has been investigated over many years. Not only is it relevant to the so-called strong $\mathrm{CP}$ problem and axion physics, it has recently gained a renewed interest in the context of possible $\mathrm{CP}$ violation in heavy ion collisions [54-56]. On

\footnotetext{
${ }^{11}$ When $K>4$ all the topologically nontrivial sectors are gone, while for $K=4$ the mass expansion in each topological sector leads to different sum rules.
} 
a practical side, lattice simulations at fixed topology suffer from large finite-volume effects and it is useful to analytically understand the topology dependence of observables $[57,58]$. Various aspects of $\theta$-dependent physics are reviewed in [59].

While $\theta$-dependence in QCD is inherently nonperturbative, it is quite difficult to simulate QCD with a nonzero $\theta$ angle on the lattice because of a severe sign problem. It is then a promising alternative to employ chiral effective theories to study topological aspects of QCD at low energy [60-63]. Studies of the $\theta$ vacuum in the $\varepsilon$-regime of chiral perturbation theory were performed by various authors $[46,64-66]$. The virtue of taking the microscopic limit is that the partition function and other various nonperturbative quantities (e.g., topological susceptibility) can be computed exactly. In this section we shall extend this analysis to the Stern phase and delineate the structure of the $\theta$ vacua, emphasizing qualitative differences from the ordinary QCD vacuum.

\subsection{Quarks in the fundamental representation}

The partition function in the presence of the $\theta$ angle admits a Fourier decomposition

$$
Z(\theta)=\sum_{Q=-\infty}^{\infty} \mathrm{e}^{i Q \theta} Z_{Q},
$$

where $Z_{Q}$ is the partition function in the sector of topological charge $Q$ with

$$
Q=\frac{g^{2}}{64 \pi^{2}} \int \mathrm{d}^{4} x \varepsilon_{\alpha \beta \gamma \delta} F_{\alpha \beta}^{a} F_{\gamma \delta}^{a} .
$$

When there are $N_{f}$ quarks in the fundamental representation, the $\theta$ angle can be transferred to the complex quark mass matrix as $M \rightarrow M \mathrm{e}^{i \theta / N_{f}}$ via an axial rotation. As is evident from (2.14), $Z^{K>4}(M)$ has no dependence on the $\theta$ angle: at leading order in the $\varepsilon$ expansion, $Z_{Q}$ with $Q \neq 0$ do not contribute and we simply have $Z(\theta)=Z_{0}$.

We now focus on the $K=4$ case, and especially $N_{f}=2$ for simplicity.

Partition function with the $\boldsymbol{\theta}$ angle. Substituting $M=m \mathrm{e}^{i \theta / 2} \mathbb{1}$ in $(2.14 \mathrm{~b})$ yields

$$
Z_{N_{f}=2}^{K=4}(m, \theta)=\int_{\mathrm{SU}(2)} \mathrm{d} U \exp \left[V_{4} m^{2}\left\{h(\operatorname{tr} U)^{2}+2 g_{1}+\left(\tilde{h} \mathrm{e}^{i \theta} \operatorname{tr}\left(U^{2}\right)+\text { h.c. }\right)+\left(g_{2} \mathrm{e}^{i \theta}+\text { h.c. }\right)\right\}\right] \text {. }
$$

Although $\theta$ dependence is strongly affected by the phases of $\tilde{h}$ and $g_{2}$ we currently lack information on their physically appropriate values. To get an idea of how $Z$ depends qualitatively on $\theta$, let us assume that $\tilde{h}$ and $g_{2}$ are both real. Then, using the identity $(\operatorname{tr} U)^{2}-\operatorname{tr}\left(U^{2}\right)=2$, one obtains

$$
\begin{aligned}
Z_{N_{f}=2}^{K=4}(m, \theta) & =\mathrm{e}^{2 V_{4} m^{2}\left[g_{1}+\left(g_{2}-2 \tilde{h}\right) \cos \theta\right]} \int_{\mathrm{SU}(2)} \mathrm{d} U \mathrm{e}^{V_{4} m^{2}(h+2 \tilde{h} \cos \theta)(\operatorname{tr} U)^{2}} \\
& =\left.\mathrm{e}^{2 V_{4} m^{2}\left(g_{1}+h+g_{2} \cos \theta\right)}\left\{I_{0}\left(\alpha_{\theta}\right)-I_{1}\left(\alpha_{\theta}\right)\right\}\right|_{\alpha_{\theta} \equiv 2 V_{4}(h+2 \tilde{h} \cos \theta) m^{2}},
\end{aligned}
$$

where in the last step we have used (A.6a) in appendix A. This is the starting point of our analysis in this subsection. We demand that $U=\mathbb{1}$ be the ground state at $\theta=0$, which 


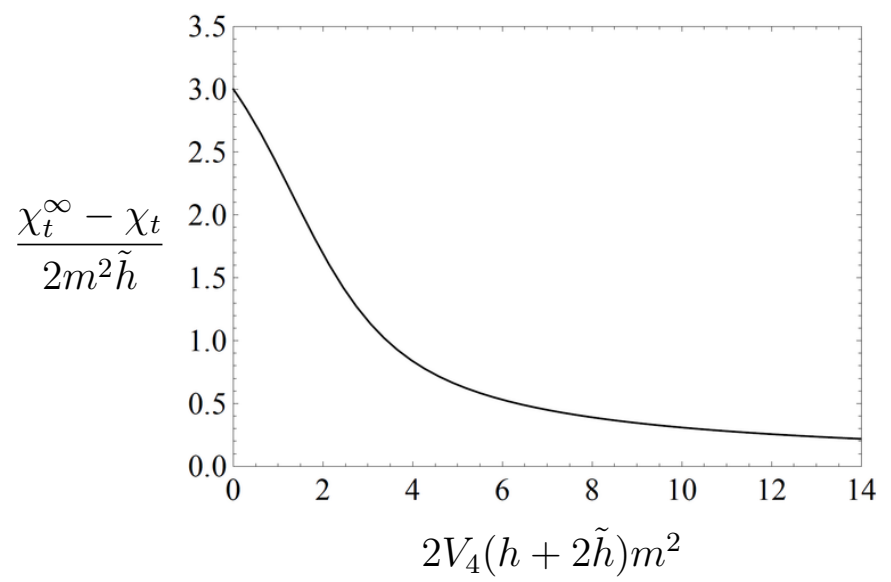

Figure 2. Convergence of the topological susceptibility $\chi_{t}$ in the microscopic limit toward $\chi_{t}^{\infty}$ for $K=4$ and $N_{f}=2$ with equal masses.

translates into the condition

$$
h+2 \tilde{h}>0 .
$$

To illustrate the physical content of (3.5) we shall calculate two quantities of major physical interest: topological susceptibility $\chi_{t}$ and the energy density $E(\theta)$.

Topological susceptibility. Let us recall the definition

$$
\chi_{t}=-\left.\frac{1}{V_{4}} \frac{\partial^{2}}{\partial \theta^{2}} \log Z\right|_{\theta=0}=\frac{\left\langle Q^{2}\right\rangle}{V_{4}} .
$$

Plugging (3.5) into this definition, we obtain

$$
\chi_{t}=\left.2 m^{2}\left\{g_{2}-\tilde{h} \frac{I_{0}\left(\alpha_{0}\right)-2 I_{1}\left(\alpha_{0}\right)+I_{2}\left(\alpha_{0}\right)}{I_{0}\left(\alpha_{0}\right)-I_{1}\left(\alpha_{0}\right)}\right\}\right|_{\alpha_{0}=2 V_{4}(h+2 \tilde{h}) m^{2}} .
$$

In particular, in the macroscopic limit $\left(\alpha_{0} \gg 1\right),{ }^{12}$ one finds $\chi_{t} \rightarrow \chi_{t}^{\infty}:=2 m^{2}\left(g_{2}+2 \tilde{h}\right)$. While it is natural that $\chi_{t}^{\infty}$ vanishes in the chiral limit, we find it interesting that $\chi_{t}^{\infty} \propto m^{2}$, in contrast to the conventional behavior $\chi_{t}=\Sigma m / N_{f} \propto m$ in the QCD vacuum [46]. Figure 2 plots the deviation of $\chi_{t}$ from $\chi_{t}^{\infty}$ as a function of the scaling parameter $\alpha_{0}$. We note that it is the combination $h+2 \tilde{h}$ that controls the finite-volume effect for $\chi_{t}$ and that $g_{1}$ and $g_{2}$ play no role here because they do not couple to the pion fluctuations.

Energy density. Next we calculate the $\theta$-dependent energy density defined by

$$
E(\theta)=-\frac{1}{V_{4}} \log Z(\theta) .
$$

Let us start with the macroscopic limit $\left(1 \ll V_{4} h m^{2} \sim V_{4} \tilde{h} m^{2}\right)$. In this limit the integral in (3.4) is dominated by contributions from saddle points. Depending on the sign of

\footnotetext{
${ }^{12}$ Recall that $\alpha_{0}>0$ due to $(3.6)$.
} 


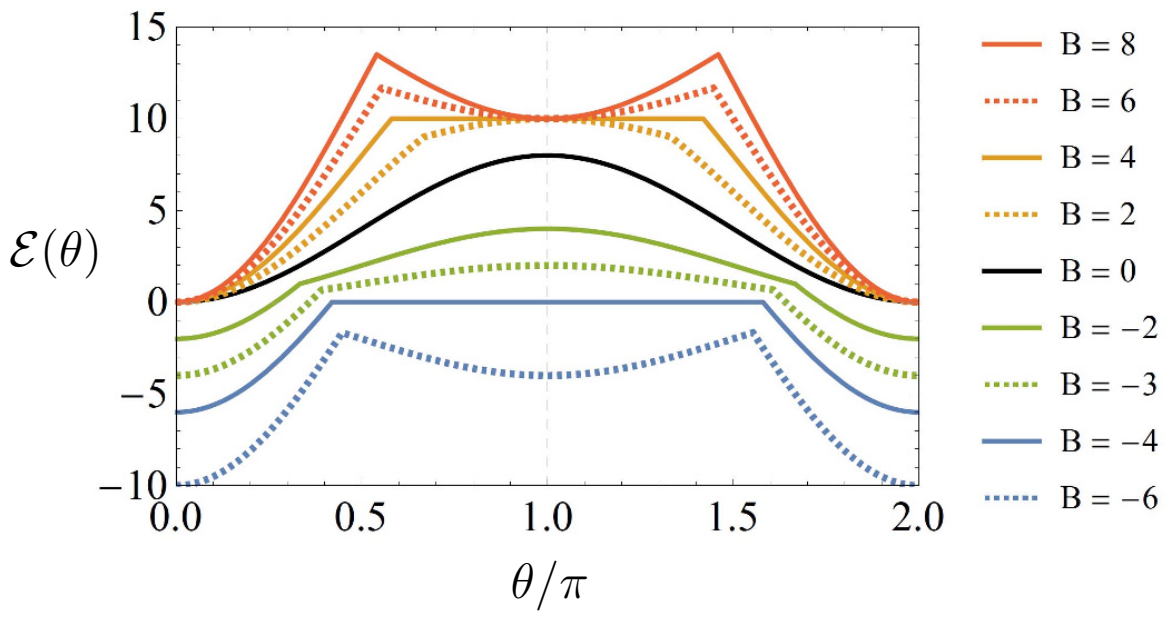

Figure 3. Dimensionless energy density (3.12) in the macroscopic limit for $K=4$ and $N_{f}=2$ with equal masses at $A \equiv g_{2} /|h|=4$ for varying $B \equiv 2 \tilde{h} / h$.

$h+2 \tilde{h} \cos \theta$, the dominant saddle corresponds to either $\operatorname{tr} U= \pm 2$ or $\operatorname{tr} U=0$. With this taken into account, we obtain

$$
\begin{aligned}
E(\theta) & =-\frac{1}{V_{4}} \log Z_{N_{f}=2}^{K=4}(m, \theta) \\
& \simeq-2 m^{2}\left(g_{1}+h+g_{2} \cos \theta+|h+2 \tilde{h} \cos \theta|\right)
\end{aligned}
$$

up to subleading corrections. This function exhibits some interesting features.

$\checkmark$ When $h>2 \tilde{h}$, it follows (recall (3.6)) that $h>2|\tilde{h}|$, so the energy becomes an analytic function of $\theta: E(\theta)=-2 m^{2}\left\{g_{1}+2 h+\left(g_{2}+2 \tilde{h}\right) \cos \theta\right\}$. No phase transition is encountered as $\theta$ is varied.

$\checkmark$ By contrast, when $h<2 \tilde{h}, E(\theta)$ becomes non-analytic at those $\theta$ where $\cos \theta=$ $-h / 2 \tilde{h}$. There are two first-order phase transitions in $0<\theta<2 \pi$.

To examine the behavior of $E(\theta)$ it is useful to define the dimensionless energy density

$$
\mathcal{E}(\theta) \equiv-A \cos \theta-|1+B \cos \theta|+1+A+B
$$

with $A \equiv g_{2} /|h|$ and $B \equiv 2 \tilde{h} / h .^{13} \mathcal{E}(\theta)$ is plotted in figure 3 for varying $B$ at $A=4$. We observe that $\mathcal{E}(\theta)$ has two cusps for all $|B|>1$. At these first-order transition points, there are two degenerate vacua with equal energy density that can coexist by forming a domain wall.

It is worth stressing that the $\theta$-dependence of the energy density presented here dramatically differs from that of QCD vacuum. In the orthodox chiral perturbation theory, a first-order transition takes place at $\theta=\pi$ for two degenerate flavors and there $\mathrm{CP}$ is

\footnotetext{
${ }^{13} \mathcal{E}(\theta)$ has the same $\theta$ dependence as $E(\theta) / 2 m^{2}|h|$. The constant part of $\mathcal{E}(\theta)$ was chosen for an aesthetic reason (to ensure that curves of $\mathcal{E}(\theta)$ for different values of $B$ in figure 3 do not intersect).
} 


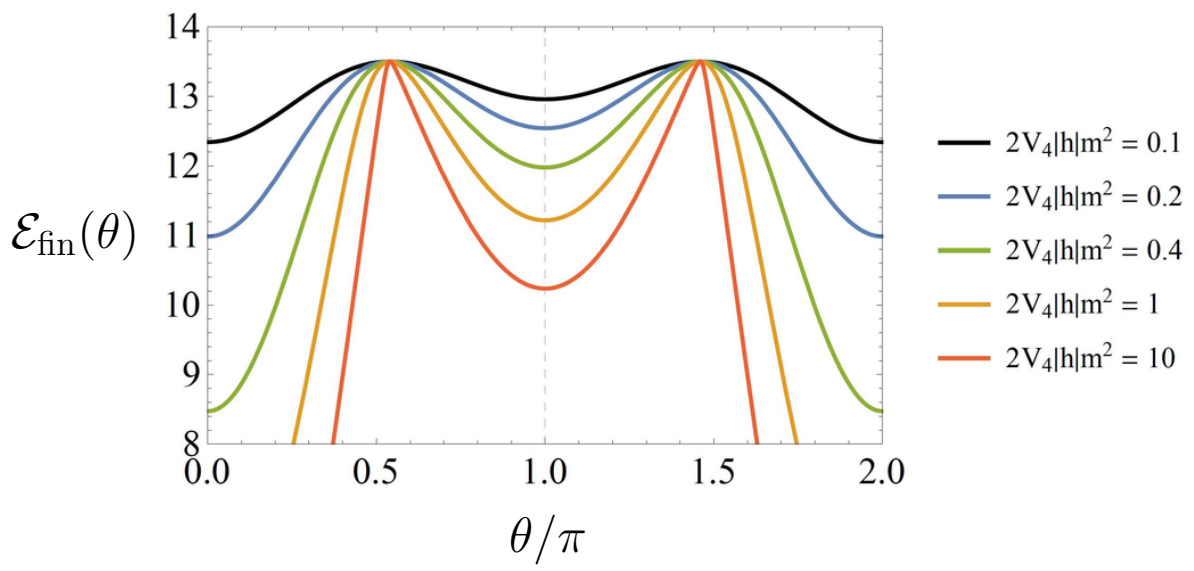

Figure 4. Finite-volume energy density (3.14) for $K=4$ and $N_{f}=2$ with equal masses for varying $2 V_{4}|h| m^{2}$ at $A=4$ and $B=8$. In the limit $2 V_{4}|h| m^{2} \rightarrow \infty$ the curves converge to the $B=8$ curve in figure 3 .

spontaneously broken $[60,61,65,67]$. By contrast, nothing dramatic happens at $\theta=\pi$ in the Stern phase.

An important remark on the topological charge distribution is in order. When $h>$ $2|\tilde{h}|, Z_{N_{f}=2}^{K=4}(m, \theta) \sim \mathrm{e}^{2 V_{4} m^{2}\left[g_{1}+2 h+\left(g_{2}+2 \tilde{h}\right) \cos \theta\right]}$ in the macroscopic limit. This means that topological charges are distributed according to the weight

$$
\frac{Z_{Q}}{Z(\theta=0)}=\exp \left[-\left\langle Q^{2}\right\rangle\right] I_{Q}\left(\left\langle Q^{2}\right\rangle\right) \sim \frac{\exp \left[-Q^{2} /\left(2\left\langle Q^{2}\right\rangle\right)\right]}{\sqrt{2 \pi\left\langle Q^{2}\right\rangle}},
$$

where $\left\langle Q^{2}\right\rangle=V_{4} \chi_{t}^{\infty}=2 V_{4} m^{2}\left(g_{2}+2 \tilde{h}\right)$ and in the second step we have used an asymptotic formula for the modified Bessel function of first kind. Equation (3.13) is thus valid for $1 \ll\left\langle Q^{2}\right\rangle$ and $Q \ll\left\langle Q^{2}\right\rangle$. Intriguingly, exactly the same functional form as (3.13) is known for the topological charge distribution in one-flavor QCD [46] and in high-temperature QCD [30, 68]; in both cases there are no massless Nambu-Goldstone modes because chiral symmetry is unbroken, and the topological charge obeys Poisson statistics. By contrast, the Stern phase do produce pions and yet exhibits the same topology dependence, which comes as a surprise.

Next we leave the macroscopic limit and proceed to the finite-volume regime where microscopic variables take $\mathcal{O}(1)$ values. This means that the zero-mode fluctuations of pions can no longer be ignored. The dimensionless finite-volume energy density can be defined, from (3.5), as

$$
\mathcal{E}_{\text {fin }}(\theta) \equiv-A \cos \theta-\frac{1}{2 V_{4}|h| m^{2}} \log \left\{I_{0}\left(\alpha_{\theta}\right)-I_{1}\left(\alpha_{\theta}\right)\right\}+1+A+B,
$$

which reduces to $\mathcal{E}(\theta)$ in $(3.12)$ as $V_{4}|h| m^{2} \rightarrow \infty$. In figure $4 \mathcal{E}_{\text {fin }}(\theta)$ is plotted for various $V_{4}|h| m^{2}$ at fixed $A$ and $B$. We observe that, while $\mathcal{E}_{\text {fin }}(\theta)$ is an analytic function of $\theta$, it gradually develops sharp peaks as $V_{4}|h| m^{2}$ is increased. In the limit $V_{4}|h| m^{2} \rightarrow \infty$ they turn into genuine first-order phase transitions, as depicted earlier in figure 3. 


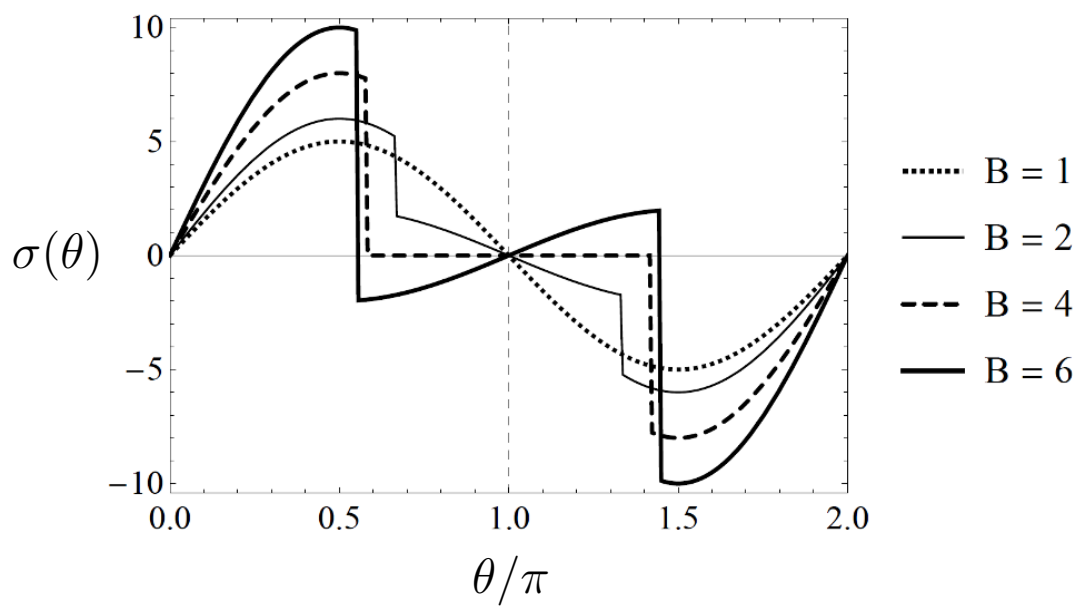

Figure 5. Topological density in the macroscopic limit for $K=4$ and $N_{f}=2$ with equal masses at $A=4$.

Topological density. The $\theta$ dependence of the vacuum can also be probed by the topological density $\left\langle\frac{g^{2}}{64 \pi^{2} i} \varepsilon_{\alpha \beta \gamma \delta} F_{\alpha \beta}^{a} F_{\gamma \delta}^{a}\right\rangle$, which is defined in a dimensionless form as

$$
\sigma(\theta) \equiv \frac{\mathrm{d} \mathcal{E}(\theta)}{\mathrm{d} \theta}
$$

in the macroscopic limit. This is plotted in figure 5. The discontinuous jumps of $\sigma(\theta)$ represent phase transitions. Intriguingly, for $A=B=4$ there is a finite range of $\theta$ where $\sigma(\theta)$ vanishes exactly. This requires fine-tuning of low-energy constants and may not be realized in the real world, though.

Exotic flavor symmetry breaking. The phase structure in the macroscopic limit is summarized in the phase diagram in figure 6 , in the plane spanned by $\theta$ and $B \equiv 2 \tilde{h} / h$. As can be seen from (3.4), phase transitions occur when $h+2 \tilde{h} \cos \theta$ switches sign. Dividing it by $h+2 \tilde{h}\left(>0\right.$; recall (3.6)) we get $\frac{1+B \cos \theta}{1+B}$, so the phase boundaries are set by $1+B \cos \theta=$ 0 and $B=-1$. In figure 6 we observe that the phase boundaries for $|B| \gg 1$ asymptote to $\theta=\pi / 2$ and $3 \pi / 2$. This is because $h+2 \tilde{h} \cos \theta \approx 2 \tilde{h} \cos \theta$ changes sign at those $\theta$ 's.

The phases in different colors in figure 6 exhibit distinctive properties.

$\checkmark$ In the white region $(h+2 \tilde{h} \cos \theta>0)$, the energy is minimized at $\operatorname{tr} U= \pm 2$, i.e., $U= \pm \mathbb{1}$. One can parametrize fluctuations around $\mathbb{1}$ as $U=\exp \left(i \phi_{a} \tau_{a} / f\right)$, insert this into (3.4) and expand the exponent up to second order in $\phi_{a}$, which enables us to read off the pion masses as

$$
m_{\pi}^{2}=\frac{8 m^{2}(h+2 \tilde{h} \cos \theta)}{f^{2}}
$$

The three pions are degenerate in this phase.

$\checkmark$ In the blue region $(h+2 \tilde{h} \cos \theta<0)$, the energy is minimized at $\operatorname{tr} U=0$ and leads to degenerate vacua. If we take $U=i \tau_{3}$ as a representative and analyze quadratic 


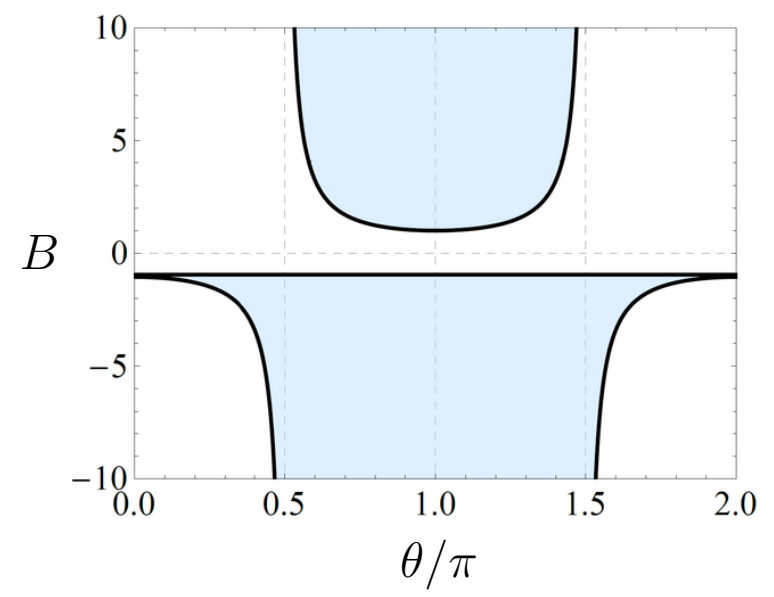

Figure 6. Phase diagram in the macroscopic limit for $K=4$ and $N_{f}=2$ with equal masses, with $B \equiv 2 \tilde{h} / h$. The energy is minimized by $\operatorname{tr} U= \pm 2$ in the empty region and by $\operatorname{tr} U=0$ in the shaded region, respectively. The phase transitions at the boundaries are generically first order.
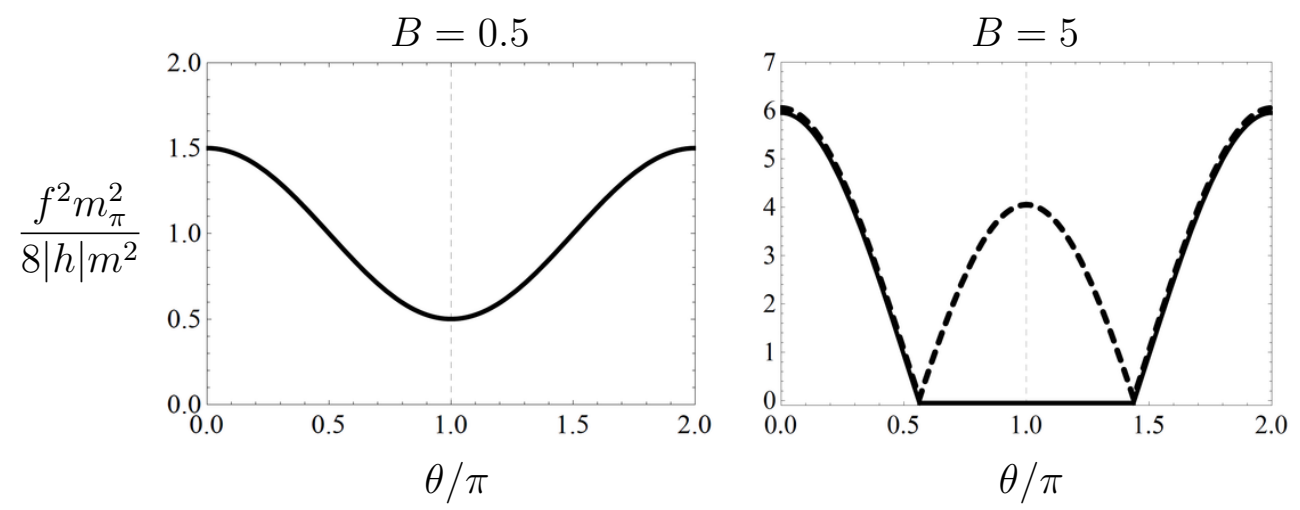

Figure 7. Pion masses as a function of $\theta$. In the left panel, all pions are degenerate. In the right panel, there are two branches that split at intermediate $\theta$. This is indicative of spontaneous breaking of $\mathrm{SU}(2)_{V}$.

fluctuations around it, the masses of three pions are found to be

$$
m_{\pi}^{2}=0,0, \text { and } \frac{8 m^{2}|h+2 \tilde{h} \cos \theta|}{f^{2}} .
$$

The two gapless modes correspond to the vector rotations in 1- and 2-directions, while the gapped mode corresponds to the axial rotation in 3-direction.

The mass spectra (3.16) and (3.17) are shown in figure 7 for $B=0.5$ and 5. At $B=5$, two of the pions go massless at intermediate $\theta$, signaling the breakdown of vectorial $\mathrm{SU}(2)$ symmetry. Since the vectorial flavor symmetry is an exact symmetry of QCD for degenerate masses, we expect that higher-order terms in the chiral effective theory would not spoil their masslessness, as long as the quark masses are degenerate. ${ }^{14}$

\footnotetext{
${ }^{14}$ Note that the Vafa-Witten theorem [69] prohibiting vectorial symmetry breaking does not apply at finite baryon density or nonzero $\theta$ angle.
} 
Two remarks on the literature are in order. First, the exotic flavor-breaking phase found above is analogous to the so-called Aoki phase [17] in lattice QCD with Wilson fermions. It has been shown by Sharpe and Singleton that the Aoki phase originates from a competition among terms at $\mathcal{O}(m), \mathcal{O}(a)$ and $\mathcal{O}\left(a^{2}\right)$ in the chiral Lagrangian [70], with $a$ the lattice spacing. This is similar in essence to our effective theory for the Stern phase, in which competing terms arise at $\mathcal{O}\left(m^{2}\right)$. Secondly, it has been pointed out by Creutz [36] and Smilga [37] for $N_{f}=2$ and $\theta \approx \pi$ that a similar vectorial flavor breaking can take place even in the standard chiral effective theory if a particular sign is chosen for a low-energy constant at $\mathcal{O}\left(p^{4}\right)$. Although their analysis has nothing to do with the Stern phase, the technical aspects of their analysis are similar to ours.

The extension of results in this section to non-degenerate masses or to $N_{f}>2$ would be technically more involved. This is deferred to future work.

\subsection{Quarks in higher representations}

In this section we consider $\theta$-dependence of QCD-like theories with $N_{f}>1$ flavors of Dirac fermions in a general complex representation $R$ of the gauge group. The motivation for such an extension comes from several directions. First, gauge theories with fermions in higher representations have attracted interests as promising candidates of the beyond-StandardModel physics [71-73]. Secondly, large- $N$ QCD with quarks in the adjoint, two-index symmetric and antisymmetric representations of the gauge group are of interest from the viewpoint of orientifold planar equivalence $[74,75]$. Of course, whether the Stern phase can be realized in such theories is a highly nontrivial dynamical question for which we have no definitive answer yet. In what follows, we shall take the existence of the Stern phase as an assumption and discuss outcomes specific to quarks in higher representation.

First and foremost, the index theorem states that $I_{R}=2 T_{R} Q$ in the background of gauge fields with the topological charge $Q$. Then the $\theta$-angle enters the partition function only through the combination $\mathrm{e}^{i \theta}(\operatorname{det} M)^{2 T_{R}}$, or in other words, the $\theta$ dependence can be incorporated into effective theory via an axial rotation

$$
M \rightarrow M \exp \left(\frac{i \theta}{2 N_{f} T_{R}}\right) .
$$

Looking back at (2.14), we again find that the case with $K>4$ has no $\theta$ dependence at leading order of the $\varepsilon$ expansion. To see $\theta$-dependent physics and for the sake of technical simplicity, we concentrate on the $N_{f}=2$ and $K=4$ case in the following. Furthermore, to make the discussion explicit, we will take $R$ to be the sextet (two-index symmetric) representation of $\mathrm{SU}(3),{ }^{15}$ for which $T_{R}=5 / 2$, although any other higher representation will do the job. From (2.4) the non-anomalous subgroup of $U(1)_{A}$ is $\mathbb{Z}_{20}$, which is supposed to be spontaneously broken to $\mathbb{Z}_{K}=\mathbb{Z}_{4}$.

Plugging $M=m \mathrm{e}^{i \theta / 10} \mathbb{1}$ into (2.14b) one finds the finite-volume partition function for sextet fermions,

$$
\begin{aligned}
Z_{N_{f}=2}^{K=4}(m, \theta)= & \int_{\mathrm{SU}(2)} \mathrm{d} U \exp \left[V _ { 4 } m ^ { 2 } \left\{h(\operatorname{tr} U)^{2}+2 g_{1}+\left(\tilde{h} \mathrm{e}^{i \theta / 5} \operatorname{tr}\left(U^{2}\right)+\text { h.c. }\right)\right.\right. \\
& \left.\left.+\left(g_{2} \mathrm{e}^{i \theta / 5}+\text { h.c. }\right)\right\}\right] .
\end{aligned}
$$

\footnotetext{
${ }^{15}$ Asymptotic freedom requires $N_{f} \leq 3$.
} 
A new interesting feature of this partition function is that it is periodic in $\theta$ with period $10 \pi$, rather than $2 \pi$. This appears to contradict the $2 \pi$-periodicity of (3.1). The resolution of this "puzzle" goes as follows. As noted above, the theory with sextet quarks has $\mathbb{Z}_{20}$ unbroken axial symmetry in the chiral limit. The putative higher-order (e.g., quartic) quark condensate is invariant only under $\mathbb{Z}_{4} \subset \mathbb{Z}_{20}$, so there are five degenerate vacua. (We remind the reader that the existence of five isolated components of the vacuum manifold follows from (2.6) in section 2.1.1.) Once we switch on the quark masses, the five-fold degeneracy is lifted and one of those vacua is selected as the unique ground state. In fact, the effective theory (3.19) is a theory of fluctuations around such a ground state. Now, if we rotate the $\theta$ angle gradually, those five vacua are permutated in a cyclic way and the ground state moves from one state to another. After a $2 \pi$ rotation of $\theta$, those five low-lying states undergo a cyclic rotation by one unit, and the system as a whole returns to itself, despite that each state returns to itself only after $10 \pi$ rotation of $\theta$.

We now have two comments:

- This mechanism was already pointed out by Leutwyler and Smilga [46] for $\mathrm{SU}(N)$ gauge theory with adjoint quarks. They explained how the $2 \pi N$-periodicity of the effective theory in $\theta$ can be reconciled with the $2 \pi$-periodicity of the full theory. As noted in [76], this also pertains to the well-known subtlety that the pure Yang-Mills partition function can be $2 \pi$-periodic in $\theta$ even though the large- $N$ scaling tells that the natural variable in the large- $N$ limit is $\theta / N$ rather than $\theta[60,77]$.

- When quarks are in the fundamental representation $\left(T_{R}=1 / 2\right)$, the vacuum manifold in the chiral limit only has a single connected component [cf. (2.6) in section 2.1.1]. This means that in the case of fundamental quarks we need not sum up contributions from multiple disconnected sectors explicitly to recover $2 \pi$-periodicity of the full partition function.

The full partition function for the Stern phase with sextet quarks may be defined as

$$
Z_{\square}(\theta):=\frac{1}{5} \sum_{k=0}^{4} Z_{N_{f}=2}^{K=4}(m, \theta+2 \pi k),
$$

which is manifestly $2 \pi$-periodic in $\theta$. If we take the macroscopic limit in the $\varepsilon$-regime, the state having the lowest energy will dominate (3.20). The energy density is therefore

$$
E_{\square}(\theta):=\min _{0 \leq k \leq 4} E\left(\frac{\theta+2 \pi k}{5}\right)
$$

with $E(\theta)$ in (3.11). The dimensionless energy density can be defined similarly as

$$
\mathcal{E}_{\square}(\theta):=\min _{0 \leq k \leq 4} \mathcal{E}\left(\frac{\theta+2 \pi k}{5}\right)
$$

with $\mathcal{E}(\theta)$ in (3.12). Figure 8 displays $\mathcal{E}_{\square}(\theta)$ for $0 \leq \theta \leq 10 \pi$ with two sets of $A$ and $B$. One can clearly observe the branched structure of the $\theta$ vacuum. In the left panel, first-order phase transitions occur at $\theta=(2 \ell+1) \pi$ with $\ell \in \mathbb{Z}$. Notably, the exotic flavor-breaking phase found for fundamental quarks in section 3.1 does not appear. This is because for $A=4$ and $B=8$ the exotic phase tends to have higher energy than the normal phase and is consequently disfavored in the minimization in (3.22). 

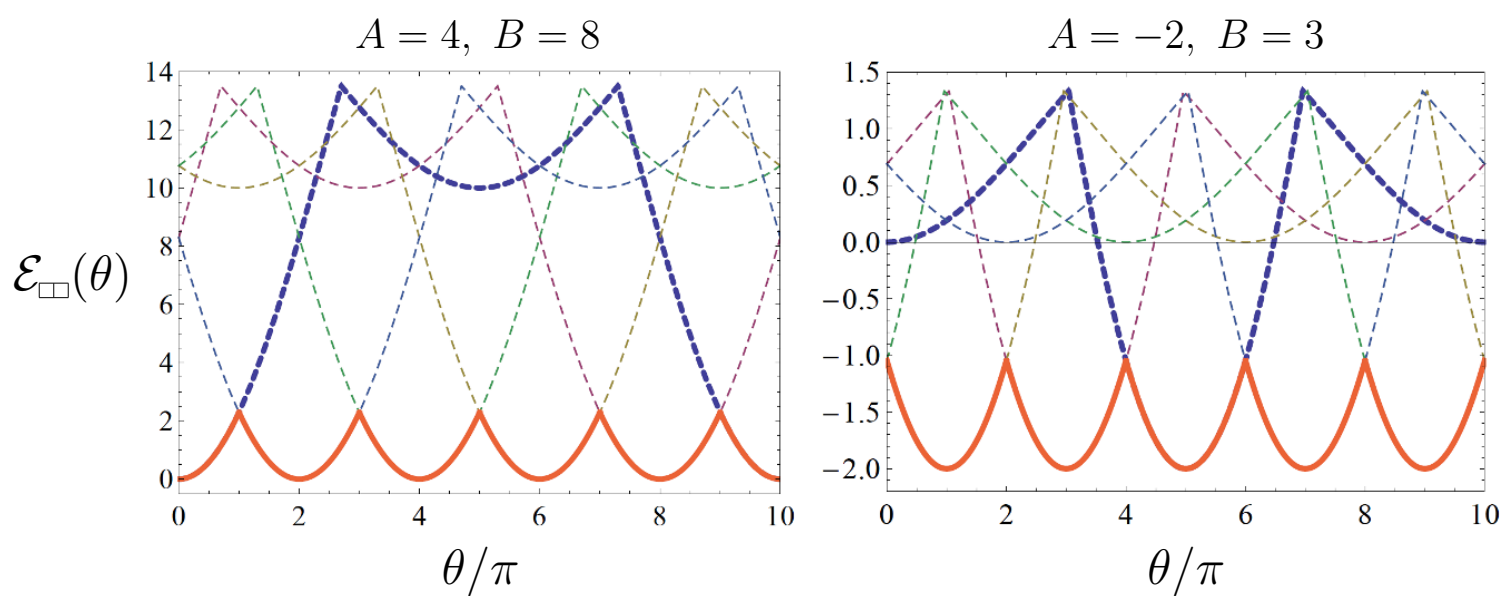

Figure 8. Dimensionless energy density (3.22) [thick solid line in orange] in the macroscopic limit for $K=4$ and $N_{f}=2$ with equal masses in the sextet representation. The $k=0$ branch is denoted by a thick blue dashed line.

However this is not necessarily true for other values of $A$ and $B$. By decreasing $A$ one can lower the energy of the exotic phase at will. As an example, we show in the right panel of figure 8 the energy density for $A=-2$ and $B=3$. In this case the first-order phase transitions occur at $\theta=2 \ell \pi$ with $\ell \in \mathbb{Z}$. At $\theta=0$ the vacuum is two-fold degenerate and breaks parity spontaneously. ${ }^{16}$ Moreover, we discover that the exotic flavor-breaking phase is realized for all values of $\theta !^{17}$ This tells us that the condition (3.6) is not sufficient, in the case of quarks in higher representations, to ensure that $U=\mathbb{1}$ is the ground state at $\theta=0$. In short, the Stern phase with quarks in higher representations exhibits an "all-or-nothing" behavior: if the vectorial flavor symmetry is unbroken at $\theta=0$, it is unbroken at any $\theta$, and conversely, if it is broken at $\theta=0$, it remains broken at any $\theta$. Since this is possible only if multiple states exchange dominance for varying $\theta$, it cannot happen for quarks in the fundamental representation that have only one vacuum sector.

\section{Conclusion}

We have investigated properties of the Stern phase using the low-energy effective theory of pions at zero and nonzero vacuum angle $\theta$. Analytical results are obtained for the $\theta$ and volume-dependence of miscellaneous physical quantities, both for fundamental quarks and for quarks in higher representations of the gauge group. We have highlighted an

\footnotetext{
${ }^{16}$ Spontaneous parity breaking at finite chemical potential is not ruled out by the Vafa-Witten theorem [78] because the path-integral measure becomes complex. We refer to [79-81] for recent model studies of paritybreaking phases at finite density. It also deserves attention that the Vafa-Witten theorem [78] can fail for quark bilinears even with positive-definite measures [17, 82]; various authors have investigated limitations of the original proof of the theorem [70, 83-87]. It would be quite interesting to extend the proof so as to incorporate four-fermion condensates, though we do not attempt it here.

${ }^{17}$ This can be checked as follows. Let us first notice that the $k=0$ branch is the ground state for $4 \pi \leq \theta \leq 6 \pi$. This implies that, for $\theta$ in this range, it is the sign of $h+2 \tilde{h} \cos \left(\frac{\theta+2 \pi k}{5}\right)=h+2 \tilde{h} \cos (\theta / 5)$ that determines whether the exotic flavor-breaking phase is realized or not. Since $B+1=(h+2 \tilde{h}) / h=$ $4>0$ we have $h>0$. Next note that $1+B \cos (\theta / 5)<0$ for $\theta \in[4 \pi, 6 \pi]$. These together imply that $h+2 \tilde{h} \cos (\theta / 5)=h\{1+B \cos (\theta / 5)\}<0$. Therefore the flavor-breaking phase with $\operatorname{tr} U=0$ is realized. Upon inspection this is seen to extend to all $\theta$.
} 
intricate interplay of multiple competing terms in the chiral Lagrangian and discussed its relevance for the phase structure of the Stern phase at nonzero $\theta$. Instead of the standard Dashen's phenomenon at $\theta=\pi$, we have found either two first-order phase transitions or no transition at all, depending on the values of low-energy constants. Throughout this work we have only relied on symmetries of the system. Therefore the obtained results should be robust as long as a nonzero mass gap exists for non-Nambu-Goldstone modes.

Since lattice simulations at finite density or $\theta \neq 0$ are currently unfeasible, it will be worthwhile to extend and improve theoretical examinations along the lines of this work further. There are several future directions. Firstly, we can generalize our analysis of the $\theta$ vacua in section 3 from $N_{f}=2$ to more flavors. Since there are more independent terms at $\mathcal{O}\left(M^{2}\right)$ than for $N_{f}=2$ we can expect richer physics. A thorough study of profiles of domain walls discussed in section 2.1.1 may also be intriguing. Throughout this work, we did not attempt to find out the microscopic mechanism that realizes the Stern phase in finite-density QCD. This is a challenging open problem that no doubt deserves further investigation. Another interesting direction is to extend the present work to QCD-like theories with quarks in (pseudo)real representations of the gauge group. These theories enjoy extended flavor symmetries and it is interesting to ask how to define the Stern phase in this case. From a phenomenological point of view it is important to incorporate the effects of isospin chemical potential into the effective theory, which can be done along the lines of $[88,89]$. Analytical calculation of the unitary integrals in (2.14) is an open mathematical problem.

\section{Acknowledgments}

This work was supported by the RIKEN iTHES project. Useful discussions with Tilo Wettig and Naoki Yamamoto are gratefully acknowledged.

\section{A Derivation of $\chi_{u d}$ in (2.24)}

This appendix outlines the derivation of (2.24). For brevity we introduce a shorthand notation for a group average:

$$
\langle\langle f(U)\rangle\rangle:=\int_{\mathrm{SU}(2)} \mathrm{d} U f(U) .
$$

Then

$$
\begin{aligned}
& \frac{\partial^{2}}{\partial m_{u} \partial m_{d}^{*}} \log \left\langle\left\langle\mathrm{e}^{V_{4} h \operatorname{tr}(M U) \operatorname{tr}\left(U^{\dagger} M^{\dagger}\right)}\right\rangle\right\rangle \\
& =V_{4} h \frac{\partial}{\partial m_{u}} \frac{\left\langle\left\langle\operatorname{tr}(M U) U_{22}^{*} \mathrm{e}^{V_{4} h \operatorname{tr}(M U) \operatorname{tr}\left(U^{\dagger} M^{\dagger}\right)}\right\rangle\right\rangle}{\left\langle\left\langle\mathrm{e}^{V_{4} h \operatorname{tr}(M U) \operatorname{tr}\left(U^{\dagger} M^{\dagger}\right)}\right\rangle\right\rangle} \\
& =V_{4} h \frac{\left\langle\left\langle U_{11} U_{22}^{*} \mathrm{e}^{V_{4} h \operatorname{tr}(M U) \operatorname{tr}\left(U^{\dagger} M^{\dagger}\right)}\right\rangle\right\rangle}{\left\langle\left\langle\mathrm{e}^{V_{4} h \operatorname{tr}(M U) \operatorname{tr}\left(U^{\dagger} M^{\dagger}\right)}\right\rangle\right\rangle}+\left(V_{4} h\right)^{2} \frac{\left\langle\left\langle U_{11} U_{22}^{*} \operatorname{tr}(M U) \operatorname{tr}\left(U^{\dagger} M^{\dagger}\right) \mathrm{e}^{V_{4} h \operatorname{tr}(M U) \operatorname{tr}\left(U^{\dagger} M^{\dagger}\right)}\right\rangle\right\rangle}{\left\langle\left\langle\mathrm{e}^{V_{4} h \operatorname{tr}(M U) \operatorname{tr}\left(U^{\dagger} M^{\dagger}\right)}\right\rangle\right\rangle} \\
& -\left(V_{4} h\right)^{2} \frac{\left\langle\left\langle\operatorname{tr}(M U) U_{22}^{*} \mathrm{e}^{V_{4} h \operatorname{tr}(M U) \operatorname{tr}\left(U^{\dagger} M^{\dagger}\right)}\right\rangle\right\rangle\left\langle\left\langle\operatorname{tr}\left(U^{\dagger} M^{\dagger}\right) U_{11} \mathrm{e}^{V_{4} h \operatorname{tr}(M U) \operatorname{tr}\left(U^{\dagger} M^{\dagger}\right)}\right\rangle\right\rangle}{\left\langle\left\langle\mathrm{e}^{V_{4} h \operatorname{tr}(M U) \operatorname{tr}\left(U^{\dagger} M^{\dagger}\right)}\right\rangle\right\rangle^{2}} .
\end{aligned}
$$


After switching to the microscopic variables $\mu_{f} \equiv 2 \sqrt{V_{4} h} m_{f}$ and taking the degenerate mass limit, we get

$$
\begin{gathered}
\frac{1}{h}\left[\lim _{m_{u, d} \rightarrow m} \frac{1}{V_{4}} \frac{\partial^{2}}{\partial m_{u} \partial m_{d}^{*}} \log \left\langle\left\langle\mathrm{e}^{V_{4} h \operatorname{tr}(M U) \operatorname{tr}\left(U^{\dagger} M^{\dagger}\right)}\right\rangle\right\rangle\right] \\
=\frac{\left\langle\left\langle U_{11} U_{22}^{*} \mathrm{e}^{\frac{\mu^{2}}{4}(\operatorname{tr} U)^{2}}\right\rangle\right\rangle}{\left\langle\left\langle\mathrm{e}^{\frac{\mu^{2}}{4}(\operatorname{tr} U)^{2}}\right\rangle\right\rangle}+\frac{\mu^{2}}{4} \frac{\left\langle\left\langle U_{11} U_{22}^{*}(\operatorname{tr} U)^{2} \mathrm{e}^{\frac{\mu^{2}}{4}(\operatorname{tr} U)^{2}}\right\rangle\right\rangle}{\left\langle\left\langle\mathrm{e}^{\frac{\mu^{2}}{4}(\operatorname{tr} U)^{2}}\right\rangle\right\rangle} \\
-\frac{\mu^{2}}{4} \frac{\left\langle\left\langle(\operatorname{tr} U) U_{22}^{*} \mathrm{e}^{\frac{\mu^{2}}{4}(\operatorname{tr} U)^{2}}\right\rangle\right\rangle\left\langle\left\langle(\operatorname{tr} U) U_{11} \mathrm{e}^{\frac{\mu^{2}}{4}(\operatorname{tr} U)^{2}}\right\rangle\right\rangle}{\left\langle\left\langle\mathrm{e}^{\frac{\mu^{2}}{4}(\operatorname{tr} U)^{2}}\right\rangle\right\rangle^{2}}
\end{gathered}
$$

To compute the group average it is convenient to adopt the parametrization based on $\mathrm{SU}(2) \cong S^{3}$ :

$$
U=\left(\begin{array}{cc}
x_{0}+i x_{3} & x_{2}+i x_{1} \\
-x_{2}+i x_{1} & x_{0}-i x_{3}
\end{array}\right) \quad \text { with } \quad x_{0}^{2}+x_{1}^{2}+x_{2}^{2}+x_{3}^{2}=1
$$

Then the followings hold for arbitrary $\alpha \in \mathbb{R}$ :

$$
\begin{aligned}
\left\langle\left\langle\mathrm{e}^{2 \alpha x_{0}^{2}}\right\rangle\right\rangle & =\mathrm{e}^{\alpha}\left\{I_{0}(\alpha)-I_{1}(\alpha)\right\}, \\
\left\langle\left\langle x_{0}^{2} \mathrm{e}^{2 \alpha x_{0}^{2}}\right\rangle\right\rangle & =\frac{1}{4} \mathrm{e}^{\alpha}\left\{I_{0}(\alpha)-I_{2}(\alpha)\right\}, \\
\left\langle\left\langle x_{3}^{2} \mathrm{e}^{2 \alpha x_{0}^{2}}\right\rangle\right\rangle & =\frac{1}{12} \mathrm{e}^{\alpha}\left\{3 I_{0}(\alpha)-4 I_{1}(\alpha)+I_{2}(\alpha)\right\}, \\
\left\langle\left\langle x_{0}^{4} \mathrm{e}^{2 \alpha x_{0}^{2}}\right\rangle\right\rangle & =\frac{1}{16} \mathrm{e}^{\alpha}\left\{2 I_{0}(\alpha)+I_{1}(\alpha)-2 I_{2}(\alpha)-I_{3}(\alpha)\right\}, \\
\left\langle\left\langle x_{0}^{2} x_{3}^{2} \mathrm{e}^{2 \alpha x_{0}^{2}}\right\rangle\right\rangle & =\frac{1}{48} \mathrm{e}^{\alpha}\left\{2 I_{0}(\alpha)-I_{1}(\alpha)-2 I_{2}(\alpha)+I_{3}(\alpha)\right\},
\end{aligned}
$$

where $I_{n}(\alpha)$ is the modified Bessel function of the first kind.

We now substitute (A.4) into (2.23) and use (A.6) with $\alpha=\mu^{2} / 2$, which yields

$$
\begin{aligned}
\frac{\chi_{u d}}{2 h} & =\frac{\left\langle\left\langle\left(x_{0}^{2}-x_{3}^{2}\right) \mathrm{e}^{\mu^{2} x_{0}^{2}}\right\rangle\right\rangle}{\left\langle\left\langle\mathrm{e}^{\mu^{2} x_{0}^{2}}\right\rangle\right\rangle}+\mu^{2} \frac{\left\langle\left\langle x_{0}^{2}\left(x_{0}^{2}-x_{3}^{2}\right) \mathrm{e}^{\mu^{2} x_{0}^{2}}\right\rangle\right\rangle}{\left\langle\left\langle\mathrm{e}^{\mu^{2} x_{0}^{2}}\right\rangle\right\rangle}-\mu^{2} \frac{\left\langle\left\langle x_{0}^{2} \mathrm{e}^{\mu^{2} x_{0}^{2}}\right\rangle\right\rangle^{2}}{\left\langle\left\langle\mathrm{e}^{\mu^{2} x_{0}^{2}}\right\rangle\right\rangle^{2}} \\
& =\frac{1}{3} \frac{I_{1}-I_{2}}{I_{0}-I_{1}}+\frac{\mu^{2}}{12} \frac{I_{0}+I_{1}-I_{2}-I_{3}}{I_{0}-I_{1}}-\frac{\mu^{2}}{16}\left(\frac{I_{0}-I_{2}}{I_{0}-I_{1}}\right)^{2},
\end{aligned}
$$

with the argument $\mu^{2} / 2$ omitted. This is the desired result.

Open Access. This article is distributed under the terms of the Creative Commons Attribution License (CC-BY 4.0), which permits any use, distribution and reproduction in any medium, provided the original author(s) and source are credited. 


\section{References}

[1] T. Banks and A. Casher, Chiral Symmetry Breaking in Confining Theories, Nucl. Phys. B 169 (1980) 103 [INSPIRE].

[2] J. Stern, Light quark masses and condensates in QCD, hep-ph/9712438 [INSPIRE].

[3] J. Stern, Two alternatives of spontaneous chiral symmetry breaking in QCD, hep-ph/9801282 [INSPIRE].

[4] I.I. Kogan, A. Kovner and M.A. Shifman, Chiral symmetry breaking without bilinear condensates, unbroken axial $Z(N)$ symmetry and exact $Q C D$ inequalities, Phys. Rev. D 59 (1999) 016001 [hep-ph/9807286] [INSPIRE].

[5] M.G. Alford, A. Schmitt, K. Rajagopal and T. Schäfer, Color superconductivity in dense quark matter, Rev. Mod. Phys. 80 (2008) 1455 [arXiv:0709.4635] [InSPIRE].

[6] R.F. Dashen, Chiral $\mathrm{SU}(3) \times \mathrm{SU}(3)$ as a symmetry of the strong interactions, Phys. Rev. 183 (1969) 1245 [INSPIRE].

[7] L. Radzihovsky, J. Park and P.B. Weichman, Superfluid transitions in bosonic atom-molecule mixtures near a Feshbach resonance, Phys. Rev. Lett. 92 (2004) 160402 [cond-mat/0312237].

[8] M.W.J. Romans, R.A. Duine, S. Sachdev and H.T.C. Stoof, Quantum Phase Transition in an Atomic Bose Gas with a Feshbach Resonance, Phys. Rev. Lett. 93 (2004) 020405 [cond-mat/0312446] [INSPIRE].

[9] M. Harada, C. Sasaki and S. Takemoto, Enhancement of quark number susceptibility with an alternative pattern of chiral symmetry breaking in dense matter, Phys. Rev. D 81 (2010) 016009 [arXiv: 0908.1361] [INSPIRE].

[10] P. Adhikari, T.D. Cohen, R.R.M. Ayyagari and M.C. Strother, On Chiral Symmetry Restoration at Finite Density in Large- $N_{c}$ QCD, Phys. Rev. C 83 (2011) 065201 [arXiv:1104.2236] [INSPIRE].

[11] Y. Hidaka, K. Kamikado, T. Kanazawa and T. Noumi, Phonons, pions and quasi-long-range order in spatially modulated chiral condensates, Phys. Rev. D 92 (2015) 034003 [arXiv: 1505.00848] [INSPIRE].

[12] K. Slagle, Y.-Z. You and C. Xu, Exotic quantum phase transitions of strongly interacting topological insulators, Phys. Rev. B 91 (2015) 115121 [arXiv:1409.7401] [INSPIRE].

[13] V. Ayyar and S. Chandrasekharan, Massive fermions without fermion bilinear condensates, Phys. Rev. D 91 (2015) 065035 [arXiv:1410.6474] [INSPIRE].

[14] K. Fukushima and T. Hatsuda, The phase diagram of dense QCD, Rept. Prog. Phys. 74 (2011) 014001 [arXiv: 1005.4814] [INSPIRE].

[15] S. Descotes-Genon and J. Stern, Finite volume analysis of $N_{f}$-induced chiral phase transitions, Phys. Rev. D 62 (2000) 054011 [hep-ph/9912234] [INSPIRE].

[16] L. Girlanda, J. Stern and P. Talavera, Eta-prime mass and chiral symmetry breaking at large- $N_{c}$ and $N_{f}$, Phys. Rev. Lett. 86 (2001) 5858 [hep-ph/0103221] [INSPIRE].

[17] S. Aoki, New Phase Structure for Lattice QCD with Wilson Fermions, Phys. Rev. D 30 (1984) 2653 [INSPIRE].

[18] G. 't Hooft, Symmetry Breaking Through Bell-Jackiw Anomalies, Phys. Rev. Lett. 37 (1976) 8 [INSPIRE]. 
[19] G. 't Hooft, Computation of the Quantum Effects Due to a Four-Dimensional Pseudoparticle, Phys. Rev. D 14 (1976) 3432 [Erratum ibid. D 18 (1978) 2199] [InSPIRE].

[20] M. Creutz, The 't Hooft vertex revisited, Annals Phys. 323 (2008) 2349 [arXiv:0711.2640] [INSPIRE].

[21] S. Vandoren and P. van Nieuwenhuizen, Lectures on instantons, arXiv:0802.1862 [INSPIRE].

[22] M. Eto, Y. Hirono and M. Nitta, Domain Walls and Vortices in Chiral Symmetry Breaking, PTEP 2014 (2014) 033B01 [arXiv: 1309.4559] [INSPIRE].

[23] E. Witten, Constraints on Supersymmetry Breaking, Nucl. Phys. B 202 (1982) 253 [INSPIRE].

[24] G. Veneziano and S. Yankielowicz, An Effective Lagrangian for the Pure $N=1$ Supersymmetric Yang-Mills Theory, Phys. Lett. B 113 (1982) 231 [INSPIRE].

[25] G.R. Dvali and M.A. Shifman, Domain walls in strongly coupled theories, Phys. Lett. B 396 (1997) 64 [Erratum ibid. B 407 (1997) 452] [hep-th/9612128] [INSPIRE].

[26] A. Kovner, M.A. Shifman and A.V. Smilga, Domain walls in supersymmetric Yang-Mills theories, Phys. Rev. D 56 (1997) 7978 [hep-th/9706089] [InSPIRE].

[27] I.I. Kogan, A. Kovner and M.A. Shifman, More on supersymmetric domain walls, $N$ counting and glued potentials, Phys. Rev. D 57 (1998) 5195 [hep-th/9712046] [inSPIRE].

[28] P. Sikivie, Of Axions, Domain Walls and the Early Universe, Phys. Rev. Lett. 48 (1982) 1156 [INSPIRE].

[29] J. Gasser and H. Leutwyler, Chiral Perturbation Theory: Expansions in the Mass of the Strange Quark, Nucl. Phys. B 250 (1985) 465 [INSPIRE].

[30] T. Kanazawa and N. Yamamoto, Quasi-instantons in QCD with chiral symmetry restoration, Phys. Rev. D 91 (2015) 105015 [arXiv:1410.3614] [INSPIRE].

[31] M. Knecht and J. Stern, Generalized chiral perturbation theory, hep-ph/9411253 [INSPIRE].

[32] M.G. Alford, K. Rajagopal and F. Wilczek, Color flavor locking and chiral symmetry breaking in high density QCD, Nucl. Phys. B 537 (1999) 443 [hep-ph/9804403] [InSPIRE].

[33] D.T. Son and M.A. Stephanov, Inverse meson mass ordering in color flavor locking phase of high density QCD, Phys. Rev. D 61 (2000) 074012 [hep-ph/9910491] [INSPIRE].

[34] D.T. Son and M.A. Stephanov, Inverse meson mass ordering in color flavor locking phase of high density QCD: Erratum, Phys. Rev. D 62 (2000) 059902 [hep-ph/0004095] [INSPIRE].

[35] T. Kanazawa, T. Wettig and N. Yamamoto, Chiral Lagrangian and spectral sum rules for dense two-color QCD, JHEP 08 (2009) 003 [arXiv: 0906.3579] [INSPIRE].

[36] M. Creutz, Quark masses and chiral symmetry, Phys. Rev. D 52 (1995) 2951 [hep-th/9505112] [INSPIRE].

[37] A.V. Smilga, $Q C D$ at $\theta \sim \pi$, Phys. Rev. D 59 (1999) 114021 [hep-ph/9805214] [InSPIRE].

[38] S. Aoki and M. Creutz, Pion Masses in Two-Flavor QCD with $\eta$ Condensation, Phys. Rev. Lett. 112 (2014) 141603 [arXiv: 1402.1837] [INSPIRE].

[39] T. Schäfer, Patterns of symmetry breaking in QCD at high baryon density, Nucl. Phys. B 575 (2000) 269 [hep-ph/9909574] [INSPIRE]. 
[40] K. Rajagopal and F. Wilczek, The condensed matter physics of QCD, hep-ph/0011333 [INSPIRE].

[41] G. 't Hooft et al., Recent Developments in Gauge Theories, Proceedings Nato Advanced Study Institute, Cargese, France, August 26 - September 8 1979, NATO Sci. Ser. B 59 (1980) 1.

[42] C.E. Detar and T. Kunihiro, Linear $\sigma$ Model With Parity Doubling, Phys. Rev. D 39 (1989) 2805 [INSPIRE].

[43] D. Jido, M. Oka and A. Hosaka, Chiral symmetry of baryons, Prog. Theor. Phys. 106 (2001) 873 [hep-ph/0110005] [INSPIRE].

[44] R.L. Jaffe, D. Pirjol and A. Scardicchio, Parity doubling among the baryons, Phys. Rept. 435 (2006) 157 [hep-ph/0602010] [INSPIRE].

[45] J. Gasser and H. Leutwyler, Thermodynamics of Chiral Symmetry, Phys. Lett. B 188 (1987) 477 [INSPIRE].

[46] H. Leutwyler and A.V. Smilga, Spectrum of Dirac operator and role of winding number in QCD, Phys. Rev. D 46 (1992) 5607 [inSPIRE].

[47] N. Yamamoto and T. Kanazawa, Dense QCD in a Finite Volume, Phys. Rev. Lett. 103 (2009) 032001 [arXiv: 0902 .4533] [INSPIRE].

[48] P. van Baal, $Q C D$ in a finite volume, hep-ph/0008206 [INSPIRE].

[49] R. Brower, P. Rossi and C.-I. Tan, The External Field Problem for QCD, Nucl. Phys. B 190 (1981) 699 [INSPIRE].

[50] A.D. Jackson, M.K. Sener and J.J.M. Verbaarschot, Finite volume partition functions and Itzykson-Zuber integrals, Phys. Lett. B 387 (1996) 355 [hep-th/9605183] [INSPIRE].

[51] A.B. Balantekin, Character expansions, Itzykson-Zuber integrals and the QCD partition function, Phys. Rev. D 62 (2000) 085017 [hep-th/0007161] [INSPIRE].

[52] T. Kanazawa, T. Wettig and N. Yamamoto, Banks-Casher-type relation for the BCS gap at high density, Eur. Phys. J. A 49 (2013) 88 [arXiv:1211.5332] [INSPIRE].

[53] T. Kanazawa, Dirac Spectra in Dense QCD, Springer Theses 124 (2013) 1, Springer, Japan.

[54] D. Kharzeev, R.D. Pisarski and M.H.G. Tytgat, Possibility of spontaneous parity violation in hot QCD, Phys. Rev. Lett. 81 (1998) 512 [hep-ph/9804221] [INSPIRE].

[55] D. Kharzeev, Parity violation in hot QCD: Why it can happen and how to look for it, Phys. Lett. B 633 (2006) 260 [hep-ph/0406125] [INSPIRE].

[56] D.E. Kharzeev, L.D. McLerran and H.J. Warringa, The Effects of topological charge change in heavy ion collisions: 'Event by event P and CP-violation', Nucl. Phys. A 803 (2008) 227 [arXiv:0711.0950] [INSPIRE].

[57] R. Brower, S. Chandrasekharan, J.W. Negele and U.J. Wiese, QCD at fixed topology, Phys. Lett. B 560 (2003) 64 [hep-lat/0302005] [INSPIRE].

[58] S. Aoki, H. Fukaya, S. Hashimoto and T. Onogi, Finite volume QCD at fixed topological charge, Phys. Rev. D 76 (2007) 054508 [arXiv:0707.0396] [INSPIRE].

[59] E. Vicari and H. Panagopoulos, Theta dependence of $\mathrm{SU}(N)$ gauge theories in the presence of a topological term, Phys. Rept. 470 (2009) 93 [arXiv:0803.1593] [INSPIRE].

[60] E. Witten, Large-N Chiral Dynamics, Annals Phys. 128 (1980) 363 [INSPIRE]. 
[61] P. Di Vecchia and G. Veneziano, Chiral Dynamics in the Large-N Limit, Nucl. Phys. B 171 (1980) 253 [INSPIRE].

[62] K. Kawarabayashi and N. Ohta, The Problem of $\eta$ in the Large-N Limit: Effective Lagrangian Approach, Nucl. Phys. B 175 (1980) 477 [INSPIRE].

[63] P. Nath and R.L. Arnowitt, The U(1) Problem: Current Algebra and the Theta Vacuum, Phys. Rev. D 23 (1981) 473 [INSPIRE].

[64] P.H. Damgaard, Topology and the Dirac operator spectrum in finite volume gauge theories, Nucl. Phys. B 556 (1999) 327 [hep-th/9903096] [INSPIRE].

[65] J. Lenaghan and T. Wilke, Mesoscopic QCD and the theta vacua, Nucl. Phys. B 624 (2002) 253 [hep-th/0108166] [INSPIRE].

[66] G. Akemann, J.T. Lenaghan and K. Splittorff, Dashen's phenomenon in gauge theories with spontaneously broken chiral symmetries, Phys. Rev. D 65 (2002) 085015 [hep-th/0110157] [INSPIRE].

[67] R.F. Dashen, Some features of chiral symmetry breaking, Phys. Rev. D 3 (1971) 1879 [INSPIRE].

[68] R.G. Edwards, U.M. Heller, J.E. Kiskis and R. Narayanan, Chiral condensate in the deconfined phase of quenched gauge theories, Phys. Rev. D 61 (2000) 074504 [hep-lat/9910041] [INSPIRE].

[69] C. Vafa and E. Witten, Restrictions on Symmetry Breaking in Vector-Like Gauge Theories, Nucl. Phys. B 234 (1984) 173 [inSPIRE].

[70] S.R. Sharpe and R.L. Singleton, Jr, Spontaneous flavor and parity breaking with Wilson fermions, Phys. Rev. D 58 (1998) 074501 [hep-lat/9804028] [INSPIRE].

[71] C.T. Hill and E.H. Simmons, Strong dynamics and electroweak symmetry breaking, Phys. Rept. 381 (2003) 235 [Erratum ibid. 390 (2004) 553] [hep-ph/0203079] [INSPIRE].

[72] D.D. Dietrich, F. Sannino and K. Tuominen, Light composite Higgs from higher representations versus electroweak precision measurements: Predictions for CERN LHC, Phys. Rev. D 72 (2005) 055001 [hep-ph/0505059] [INSPIRE].

[73] D.D. Dietrich and F. Sannino, Conformal window of $\mathrm{SU}(N)$ gauge theories with fermions in higher dimensional representations, Phys. Rev. D 75 (2007) 085018 [hep-ph/0611341] [INSPIRE].

[74] A. Armoni, M. Shifman and G. Veneziano, SUSY relics in one flavor QCD from a new $1 / N$ expansion, Phys. Rev. Lett. 91 (2003) 191601 [hep-th/0307097] [INSPIRE].

[75] A. Armoni, M. Shifman and G. Veneziano, From superYang-Mills theory to QCD: Planar equivalence and its implications, hep-th/0403071.

[76] R. Kaiser and H. Leutwyler, Large- $N_{c}$ in chiral perturbation theory, Eur. Phys. J. C 17 (2000) 623 [hep-ph/0007101] [INSPIRE].

[77] E. Witten, Theta dependence in the large- $N$ limit of four-dimensional gauge theories, Phys. Rev. Lett. 81 (1998) 2862 [hep-th/9807109] [INSPIRE].

[78] C. Vafa and E. Witten, Parity Conservation in QCD, Phys. Rev. Lett. 53 (1984) 535 [INSPIRE].

[79] A.A. Andrianov and D. Espriu, On the possibility of P-violation at finite baryon-number densities, Phys. Lett. B 663 (2008) 450 [arXiv:0709.0049] [INSPIRE]. 
[80] A.A. Andrianov, V.A. Andrianov and D. Espriu, Spontaneous P-violation in QCD in extreme conditions, Phys. Lett. B 678 (2009) 416 [arXiv:0904.0413] [InSPIRE].

[81] A.A. Andrianov, D. Espriu and X. Planells, Chemical potentials and parity breaking: the Nambu-Jona-Lasinio model, Eur. Phys. J. C 74 (2014) 2776 [arXiv:1310.4416] [INSPIRE].

[82] D.T. Son and M.A. Stephanov, QCD at finite isospin density, Phys. Rev. Lett. 86 (2001) 592 [hep-ph/0005225] [INSPIRE].

[83] V. Azcoiti and A. Galante, Parity and CT realization in QCD, Phys. Rev. Lett. 83 (1999) 1518 [hep-th/9901068] [INSPIRE].

[84] T.D. Cohen, Spontaneous parity violation in QCD at finite temperature: On the Inapplicability of the Vafa-Witten theorem, Phys. Rev. D 64 (2001) 047704 [hep-th/0101197] [INSPIRE].

[85] X.-d. Ji, Validity of the Vafa-Witten proof on absence of spontaneous parity breaking in QCD, Phys. Lett. B 554 (2003) 33 [hep-ph/0108162] [INSPIRE].

[86] M.B. Einhorn and J. Wudka, On the Vafa-Witten theorem on spontaneous breaking of parity, Phys. Rev. D 67 (2003) 045004 [hep-ph/0205346] [INSPIRE].

[87] V. Azcoiti, G. di Carlo and A. Vaquero, Parity realization in Vector-like theories from Fermion Bilinears, JHEP 04 (2008) 035 [arXiv: 0804.1338] [INSPIRE].

[88] M.A. Metlitski and A.R. Zhitnitsky, Theta-parameter in 2 color QCD at finite baryon and isospin density, Nucl. Phys. B 731 (2005) 309 [hep-ph/0508004] [INSPIRE].

[89] M.A. Metlitski and A.R. Zhitnitsky, theta-dependence of QCD at finite isospin density, Phys. Lett. B 633 (2006) 721 [hep-ph/0510162] [INSPIRE]. 